\title{
Adaptive Transmission Techniques for the Internet of Things
}

by

\author{
Chowdhury Saleha Ferdowsy
}

\author{
A thesis submitted to the \\ Faculty of Graduate and Postdoctoral Affairs \\ in partial fulfilment of the requirements for the degree of \\ Master of Applied Science in Electrical and Computer Engineering
}

Ottawa-Carleton Institute for Electrical and Computer Engineering

Department of Systems and Computer Engineering

Carleton University

Ottawa, Ontario, Canada

June, 2020

Copyright (C)

Chowdhury Saleha Ferdowsy, 2020 
The undersigned recommend to the Faculty of Graduate Studies and Research acceptance of the Dissertation

\title{
Adaptive Transmission Techniques for the Internet of Things
}

\author{
Submitted by Chowdhury Saleha Ferdowsy \\ in partial fulfilment of the requirements for the degree of \\ Master of Applied Science
}

Mohammed Ibnkahla, Supervisor

Department Chair

Carleton University

2020 


\section{Abstract}

The growing popularity of the Internet of Things (IoT) brings up new challenges to the field of wireless communications. Indeed, connecting numerous IoT devices from different sources of information with diverse Quality of Service (QoS) requirements leads to the need for new transmission techniques. These IoT devices are also heterogeneous in terms of several properties including their power capabilities. The design of most existing transmission techniques is done without considering this heterogeneity. In this thesis, we design and propose two sets of transmission schemes that take into consideration different properties of IoT devices, traffic type, and the heterogeneous requirements of IoT applications. The first set of adaptive transmission schemes takes into consideration the remaining battery of each IoT sensor node to decide on the transmit power and the modulation mode to be used during each transmission slot. In this context, a hybrid scheme is proposed as a throughput-energy trade-off between a spectral efficient scheme and a power saving scheme. The modes of operation of these three schemes and their performances in terms of energy consumption, spectral efficiency, and delay are presented in Chapter 3 of this thesis both in terms of simulations and analysis. The second set of transmission schemes take into consideration the heterogeneity in IoT traffic. We consider a Massive Multiple-

Input Multiple-Output (MIMO) system, in which base station (BS) is equipped with a large number of antennas serving a high number of IoT nodes from different traffic 
types and we aim to adapt and optimize the system and transmissions parameters for each of these traffics. The proposed set of schemes considers different IoT traffic's QoS requirements and based on that an energy efficient optimization problem is formulated to find the optimal number of antenna, power allocation, and modulation mode. The optimization model simultaneously determines the optimal number of antennas that should be scaled with the number of connected nodes to maximize energy efficiency (EE) of the overall system and decide modulation mode based on the traffic type. Simulations are done using MATLAB and the results show that the $\mathrm{EE}$, the average transmit power, and the area throughput improves when more nodes access the network. The proposed traffic-oriented transmission schemes are presented in Chapter 4. The results presented in this chapter show that the adaptation of the number of antennas with the proper modulation mode allows us to simultaneously accommodate heterogeneous IoT traffics in a common wireless network. 


\section{Dedicated to my beloved family.}




\section{Acknowledgments}

I would like to express my special gratitude to my supervisor, Prof. Mohamed Ibnkahla, who gave me the opportunity to become a part of the Sensor Systems and Internet of Things laboratory. I am extremely grateful to him for his excellent supervision and for providing me academic and generous financial support throughout the road to finish this thesis. I would also like to extend my warm thanks to Dr. Zied Bouida for his excellent co-supervision. His suggestions, availability, and advice have been very valuable throughout my research.

My utmost thanks go to my colleagues in the Lab for providing an engaging research environment. Special thanks go to Yousef Rafique and Abdallah Jarwan for their great support during my research. My heartfelt appreciation goes to my friend Mohammed Abuibaid who guided me in my research area and advised me with his expertise and insights. From all of them, I have learnt not only professional knowledge, but also methodologies of scientific research as well as analytical and critical thinking. All these, for sure, will benefit me throughout my life and career.

Finally, my sincere appreciation goes to my parents and husband for their constant care, love, and prayers. Most importantly, my praise and thanks is to almighty Allah, who blessed me with the strength to complete this research. Also many thanks to the Bangladeshi community for their support and encouragement towards the road of starting graduate studies in Canada. 


\section{Table of Contents}

Abstract $\quad$ iii

Acknowledgments $\quad$ vi

Table of Contents $\quad$ vii

List of Tables $\quad$ x

List of Figures $\quad$ xi

Nomenclature $\quad$ xiii

1 Introduction 1

1.1 Motivation . . . . . . . . . . . . . . . . . . 2

1.2 Thesis Contribution . . . . . . . . . . . . . . . 4

1.3 Thesis Overview . . . . . . . . . . . . . . . . 5

2 Background and Related Work $\quad 6$

2.1 Introduction to Internet of Things . . . . . . . . . . . . . . . . 6

2.1.1 Leading Domain of IoT Applications . . . . . . . . . . . . . . 7

2.1.2 Diversity of QoS in IoT Applications . . . . . . . . . . 8

2.2 Background of Massive MIMO . . . . . . . . . . . . . . . . . . 11

2.2.1 MIMO Review . . . . . . . . . . . . . . . . . 11 
2.2.2 Massive MIMO: A Scalable Technology . . . . . . . . . . . . 13

2.3 Massive MIMO for IoT . . . . . . . . . . . . . . . . . . . . 18

2.3.1 System Model Description of Massive MIMO . . . . . . . . . 18

2.3.2 Uplink Transmission . . . . . . . . . . . . . . . . . . . . 20

2.3.3 Downlink Transmission . . . . . . . . . . . . . . . . . . . . 23

2.3.4 Potential of Massive MIMO for IoT . . . . . . . . . . 25

2.4 Related Work . . . . . . . . . . . . . . . . . . . . . . . 26

2.4.1 Adaptive Transmission . . . . . . . . . . . . . . . . . 27

2.4 .2 Massive MIMO . . . . . . . . . . . . . . . . . . 28

2.5 Conclusion . . . . . . . . . . . . . . . . . . . . . . . . . . . . . . 29

3 Adaptive Transmission for Heterogeneous IoT Sensor Nodes 30

3.1 Introduction . . . . . . . . . . . . . . . . . . . . . 30

3.2 System and Channel Models . . . . . . . . . . . . . . . . . . . . 31

3.2 .1 System model . . . . . . . . . . . . . . . . . . . . . . . . 31

3.2 .2 Channel Model . . . . . . . . . . . . . . . . . . . . . . 33

3.3 Modes of Operation . . . . . . . . . . . . . . . . . . . . . . . . 34

3.3.1 Spectral Efficient Scheme (SES) . . . . . . . . . . . . . 34

3.3.2 Power Saving Scheme (PSS) _ . . . . . . . . . . . . . 35

3.3 .3 Hybrid Scheme $(\mathrm{HS}) \ldots \ldots \ldots$

3.4 Performance Evaluation $\ldots \ldots \ldots \ldots \ldots$

3.4 .1 Delay Performance . . . . . . . . . . . . . . . . . . . 38

3.4 .2 Average Spectral Efficiency _. . . . . . . . . . . 38

3.4 .3 Power Usage Ratio . . . . . . . . . . . . . . . . . . . . . . . . 40

3.5 Numerical Results . . . . . . . . . . . . . . . . . . . . . . . . . . . . . 41

3.5.1 Data Buffering . . . . . . . . . . . . . . . . . . . 42 
3.5.2 Power Usage Ratio . . . . . . . . . . . . . . . . . . . . . 43

3.5.3 Average Spectral Efficiency _. . . . . . . . . . . . . . 44

3.6 Conclusion . . . . . . . . . . . . . . . . . . . . . . . . 45

4 Traffic Adaptive Transmission Schemes for Massive MIMO based IoT network $\quad 46$

4.1 Introduction . . . . . . . . . . . . . . . . . . . 46

4.2 System Model and Assumptions . . . . . . . . . . . . . . . . 48

4.2.1 Channel Model and Linear Processing . . . . . . . . . . . . 49

$4.2 .2 \quad$ Achievable Rate . . . . . . . . . . . . . . . 50

4.2 .3 Transmit Power . . . . . . . . . . . . . . 50

$4.2 .4 \quad$ Circuit Power . . . . . . . . . . . . . . . . 51

4.3 Traffic-Oriented Transmission Scheme . . . . . . . . . . . . . . . . . 53

4.4 Numerical results . . . . . . . . . . . . . . . . . . . . . . . . . 57

4.4 .1 Optimal Number of Antenna . . . . . . . . . . . . . 58

4.4 .2 Average Transmit Power . . . . . . . . . . . . . . . . 59

$4.4 .3 \quad$ Spectral Efficiency . . . . . . . . . . . . . . . . . 61

4.4 .4 Area Throughput . . . . . . . . . . . . . . . . . . . 62

4.4 .5 Energy Efficiency . . . . . . . . . . . . . . . . . . . 64

4.5 Conclusion . . . . . . . . . . . . . . . . . . 65

5 Conclusions and Future Work $\quad 66$

5.1 Conclusions . . . . . . . . . . . . . . . . 66

5.2 Future Work . . . . . . . . . . . . . . . . . . . . . 68

$\begin{array}{ll}\text { List of Publications } & \mathbf{7 0}\end{array}$

$\begin{array}{ll}\text { List of References } & 71\end{array}$ 


\section{List of Tables}

4.1 Reference Parameter . . . . . . . . . . . . . . . . . . . 53

4.2 Simulation Parameter . . . . . . . . . . . . . . . . 58 


\section{List of Figures}

2.1 Examples of some leading IoT application domains $\ldots \ldots \ldots$

2.2 IoT applications under three service categories of $5 \mathrm{G} \ldots \ldots \ldots$

2.3 Examples of MIMO technology used in our everyday life . . . . . . 11

2.4 Single-user MIMO and multi-user MIMO. . . . . . . . . . . . . 12

2.5 Massive MIMO : a multi-user MIMO technology . . . . . . . . . 15

2.6 Illustration of TDD operation in a single coherence block. . . . . . . 20

2.7 UL operation of Massive MIMO . . . . . . . . . . . . . . . 20

2.8 DL operation of Massive MIMO . . . . . . . . . . . . . . . . . 23

3.1 System Model . . . . . . . . . . . . . . . . . . . . . . . . . 32

3.2 Hybrid scheme's mode of operation . . . . . . . . . . . 37

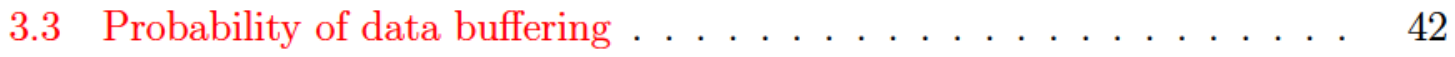

3.4 Average Power Consumption: Ratio of power used by the maximum

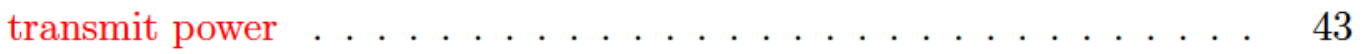

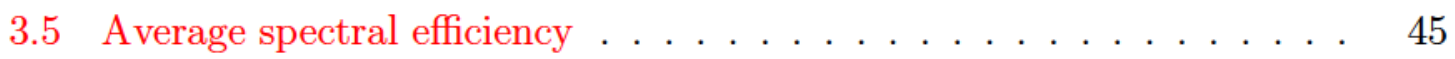

4.1 Single-cell IoT network with large number of nodes generating different

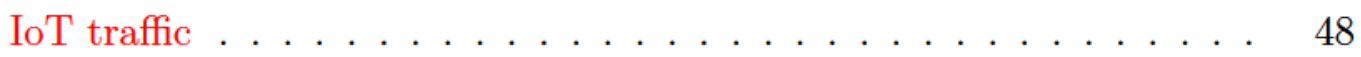

4.2 Operational diagram of traffic adaptive transmission scheme. . . . . 54

4.3 Optimal number of antennas at EE maximizing point for different traffics. 59

4.4 Average transmit power per node at EE-maximization solution. . . . 60 
4.5 Achievable spectral efficiency for different IoT traffic. . . . . . . . . . 61

4.6 Area throughput at EE maximizing solution. . . . . . . . . . . . . . . 62

4.7 Maximum energy efficiency using optimal number of antennas. . . . . 64 


\title{
Nomenclature
}

\author{
Notation Description \\ 4G Fourth Genaration \\ 5G Fifth Generation \\ ASE Average Spectral Efficiency \\ AWGN Additive White Gaussian Noise \\ BER Bit Error Rate \\ BS Base Station \\ BPSK Binary Phase Shift Keying \\ CP Circuit Power \\ CSI Channel State Information \\ DPC Dirty Paper Coding \\ DL Downlink \\ EE $\quad$ Energy Efficiency \\ eMBB Enhanced Mobile Broadband
}




\begin{tabular}{|c|c|}
\hline FDD & Frequency Division Multiplexing \\
\hline HP & High Power \\
\hline HS & Hybrid Scheme \\
\hline ICT & Information and Communication Technology \\
\hline IEEE & Institute of Electrical and Electronics Engineers \\
\hline IoT & Internet of Things \\
\hline ITU & International Telecommunication Union \\
\hline LP & Low Power \\
\hline LTE & Long Term Evolution \\
\hline MF & Matched Filter \\
\hline MIMO & Multiple Input Multiple Output \\
\hline mMTC & Massive Machine Type Communication \\
\hline MRT & Maximum Ratio Transmission \\
\hline MRC & Maximum Ratio Combining \\
\hline MU & Multi User \\
\hline NGNs & Next Generation Networks \\
\hline PSS & Power Saving Scheme \\
\hline PUR & Power Usage Ratio \\
\hline
\end{tabular}




$\begin{array}{ll}\text { QAM } & \text { Quadrature Amplitude Modulation } \\ \text { QoS } & \text { Quality of Service } \\ \text { QPSK } & \text { Quadrature Phase Shift Keying } \\ \text { SDMA } & \text { Space Division Multiple Access } \\ \text { SE } & \text { Spectral Efficiency } \\ \text { SES } & \text { Spectrally Efficient Scheme } \\ \text { SINR } & \text { Signal-to-Interference-and-Noise Ratio } \\ \text { SNR } & \text { Signal-to-Noise Ratio } \\ \text { SU } & \text { Single User } \\ \text { TDD } & \text { Time Division Duplex } \\ \text { UE } & \text { User Equipment } \\ \text { UL } & \text { Uplink } \\ \text { ZF } & \text { Zero Forcing }\end{array}$




\section{Chapter 1}

\section{Introduction}

In Internet of Things (IoT) systems, wide area coverage and salable connectivity with proper resource allocation is believed to be one of the most crucial problems [1,2]. IoT is currently connecting tens of billions of devices to communicate by means of several transmission technologies $[3,4]$. Such a wide, heterogeneous and scalable concept of IoT leads to lots of challenges in terms of physical layer connectivity. Keeping pace with higher and heterogeneous Quality of Service (QoS) demands will require new technologies that can offer acceptable communication. Therefore, there is a need for a versatile physical transmission infrastructure that can effectively support all types of traffic.

The use of adaptive transmission techniques helps improving the performance of wireless communication by taking advantage of the wireless channel varying conditions. In this context, we aim at using power and rate adaption in order to improve the performance of IoT systems. Adaptive modulation (AM) is an attractive technology to improve the transmission efficiency over wireless channels with guaranteed reliability. It yields significant performance enhancements compared to fixed modulation schemes and have been efficiently implemented in several standard wireless networks, e.g. IEEE802.11, IEEE802.16. Based on multiple thresholds, AM achieves 
high spectral efficiency (SE) in wireless communication systems. The key idea of AM is to adapt the modulation parameters, such as constellation size, to the fading channel conditions while respecting the average bit error rate (ABER) requirements. Similarly power adaptation allows to adapt the transmit power of IoT devices based on the channel conditions and power considerations that we will detail in this thesis.

Massive Multiple Input Multiple Output (Massive MIMO) is another performance enhancing technology for future wireless network. It is a multi-user MIMO technology that allows to serve multiple users simultaneously which can significantly improves SE and energy efficiency (EE). The technology of MIMO capitalizes three key methods, which are spatial diversity, spatial multiplexing, and beamforming. The first method, spatial diversity improves the reliability of wireless transmission by sending the same data across different propagation, or spatial, paths. Spatial multiplexing, the second technique aims at transmitting multiple messages simultaneously without interfering with one another as they are separated by space. Finally, the beamforming method utilizes advanced antenna technologies on both devices and networks' base stations to focus a wireless signal in a specific direction, rather than spreading the signal to a wide area. The use of aggressive spatial multiplexing by deploying a large number of antennas at BS results in dramatic improvement in capacity and EE. High spectral and energy efficiency in combination with multiple user access makes Massive MIMO one of the successful technologies that needs to be leveraged for serving heterogeneous IoT applications.

\subsection{Motivation}

To realize the full potential of IoT, several technical challenges need to be addressed which include resource limitations, heterogeneity, and massive connectivity. IoT needs 
to support diverse application scenarios, which typically have different service requirements. Also, the type of IoT devices are heterogeneous in terms of power supply, demand and communication requirement. For example, most IoT applications entails quick information exchanges from low-complexity low power smart devices/sensors. These terminals will access the network for short packet transmission with low data rate that stems from metering data, status information, remote commands and so on. The peak rate requirement of these type of terminal will have much lower than conventional traffics, such as phone calls and video streaming. The traditional cellular systems are designed for human-type communications and does not take this device and service heterogeneity into consideration. As the number of connected devices and sensors within the IoT will be enormous, the overall energy consumption of future IoT could be prohibitive with conventional transmission system if it ignores the diversity of nodes and services. As such, there is a pressing need to optimally design the transmission strategies from the perspective of individual application requirements.

To tailor IoT networks towards specific application requirements, advanced network layer approach such as network slicing [5,6] has been evolved that virtually slice the IoT network based on similar performance requirement. These network virtualization technologies enable customized services for different IoT applications operating on top of a shared physical infrastructure. Therefore, physical transmission systems of IoT must have the capability to support all the slices from diverse source of applications $[7,8]$. In this context, massive MIMO is a game changing physical layer transmission technology with regard to theory, systems, and implementation. It can offer enhanced broadband services and can be tailored to support a massive number of low power devices. Also, it is an excellent candidate to realize highly reliable communication as it can establish very robust physical links. 


\subsection{Thesis Contribution}

This thesis provides two sets of adaptive transmission schemes to address the power heterogeneity and traffic heterogeneity challenges in IoT systems. In light of the above, the main contributions of this thesis are summarised as follows:

- We studied adaptive rate and power control technique and developed an energy aware hybrid scheme for heterogeneous IoT nodes. The scheme combines two modes of operation: a) spectral efficient scheme (SES) and b) power saving scheme (PSS). In particular, we have classified IoT nodes with two categories. The node that has low remaining energy will be defined as Low Power (LP) node and the node that has high remaining energy or has a continuous power supply will be considered as High Power (HP) node. The transmission scheme determines the mode of operation based on the energy level of the connected node and adapt transmit power and rate under the prevailing channel condition.

- Selected numerical examples confirmed by analytical results, show that our proposed scheme can mitigate the trade-off between existing SES and PSS schemes by categorizing the nodes and applying proper power management.

- We then propose a novel traffic-oriented energy efficient transmission technique for Massive MIMO enabled IoT system that deals with different traffic with different QoS constraints. Based on the diverse QoS requirement, we have classified IoT traffic in three main categories (i) energy sensitive, (ii) throughput sensitive, and (iii) highly reliable traffic. We then jointly consider the uplink (UL) and downlink (DL) transmission for each traffic and our energy efficient model dynamically assign the number of antennas that should be scaled with the number of IoT nodes. After that, our transmission scheme finds the modulation mode that needs to be adapted to individual traffic. 
- We adopt the gradient decent and exhaustive search method to solve the aforementioned optimization problem. For each type of IoT traffic, the optimization process is performed with respect to the number of IoT node.

- The optimization model reveals the optimal antenna-node ratio for every type of traffic that is needed to satisfy their QoS requirement while maximizing their EE. More specifically, when a certain number of IoT nodes from a particular traffic access the network, our optimization problem aims to find the optimal number of active antennas with proper power allocation which will allow to maintain the corresponding traffic's QoS and ensure EE of the network. Simulations are performed to demonstrate the performance of the proposed energy efficient traffic-oriented transmission scheme. The results show that our proposed set of schemes provide scalability in terms of EE, area throughput, and average transmit power.

\subsection{Thesis Overview}

The outline of this thesis is as follows. Chapter 2 provides a brief background of IoT as well as massive MIMO. It then discusses incorporation of the massive MIMO technology into an IoT system. In Chapter 3, we consider a hybrid transmission scheme for a single antenna system that combines rate adaptation and power adaptation to address the power heterogeneity issue in IoT system. Motivated by this work, we proposed an energy efficient traffic oriented transmission design for massive MIMO system in Chapter 4 that considers the service heterogeneity of IoT traffic. Finally, Chapter 5 concludes this thesis along with recommendations for future work. 


\section{Chapter 2}

\section{Background and Related Work}

In this chapter, we provide a brief background of IoT, its service-centric QoS requirements, and an overview of Massive MIMO. Since the concept of Massive MIMO is an extension of MIMO, it is important to understand different notions related to MIMO technology. To that end, Section 2.1 first discusses the concept of IoT and its QoS diversity. Section 2.2 reviews the background of MIMO technology and then introduces the concept of massive MIMO. Section 2.3 discusses the system model of massive MIMO along with the performance in UL and DL transmission. It also discusses how incorporating massive MIMO into IoT can be one of the solutions to address the heterogeneity issue. Section 2.4 reviews the related work on adaptive transmission and massive MIMO. Finally, we summarize the chapter in section 2.5.

\subsection{Introduction to Internet of Things}

IoT is a network of computing devices embedded with electronics, software, sensors, and network connectivity that enables them to gather information and to perform specific actions. The evolution of IoT is the result of the convergence of multiple technologies, including advanced wireless communication, real-time analytic, machine 
learning, data analysis, and embedded systems. In this thesis, we have chosen to follow the definition of IoT proposed by the International Telecommunication Union (ITU)'s Telecommunication Standardization Sector (a United Nations agency which specializes in Information and Communication Technology (ICT)): "IoT is a global infrastructure for the information society, enabling advanced services by interconnecting (physical and virtual) things based on existing and evolving inter-operable information and communication technologies" [9]. The basic concept of IoT is to connect things together, thus allowing these "things" to communicate with each other and enable people to communicate with them.

\subsubsection{Leading Domain of IoT Applications}

Over the last few years, IoT devices are being developed and evolving at a spectacular rate bringing us closer to connected and more intelligent networks. IoT extends the Internet to our daily objects, such as appliances, cameras, lights, displays, and vehicles, by equipping them with micro-controllers, communication, and networking capabilities. Such extension will transform our daily life and will enable many new ap-

plications such as health, smart homes, agriculture, environment monitoring, industry and automation, smart cities, security and emergency, smart grid, online shopping, virtual reality, wearable technology, and intelligent transportation system as shown in Fig. 2.1. Since IoT applications are heterogeneous, their QoS requirements for transmitting data are also diverse. In addition to that, to support IoT systems, next generation networks (NGNs) should be scalable enough and should have efficient enhancements compared to the current ones in terms of speed, latency, reliability and energy efficiency $[1,8,10]$. Therefore, a flexible physical transmission infrastructure is needed to effectively support all applications and the corresponding service types. 


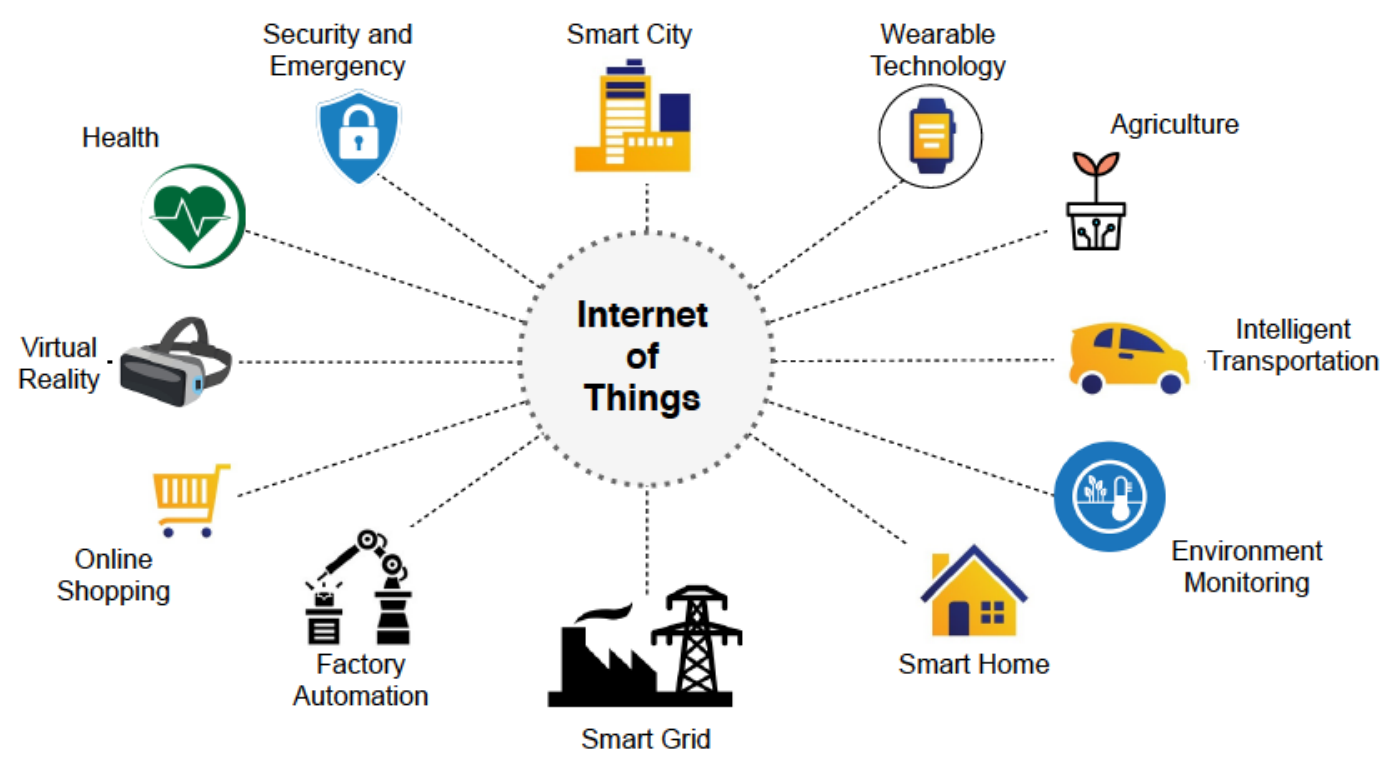

Figure 2.1: Examples of some leading IoT application domains

\subsubsection{Diversity of QoS in IoT Applications}

The service development of next generation wireless networks based on $5 \mathrm{G}$ is driven by IoT connectivity. In the context of new $5 \mathrm{G}$ use cases, the ITU has classified 5G services into three categories: (i) ultra-reliable and low latency communication (URLLC), (ii) enhanced mobile broadband (eMBB) and (iii) massive machine-type communication (mMTC) as shown in Fig. 2.2 [11]. This different service categories bring about diversified performance requirement in terms of energy efficiency, spectral efficiency, delay and reliability. The description of each service category with their corresponding QoS requirements are explained below. However, some of the applications presented in Fig. 2.2 may require more than one service for example, application of e-health will require the service of both URLLC and eMBB. 


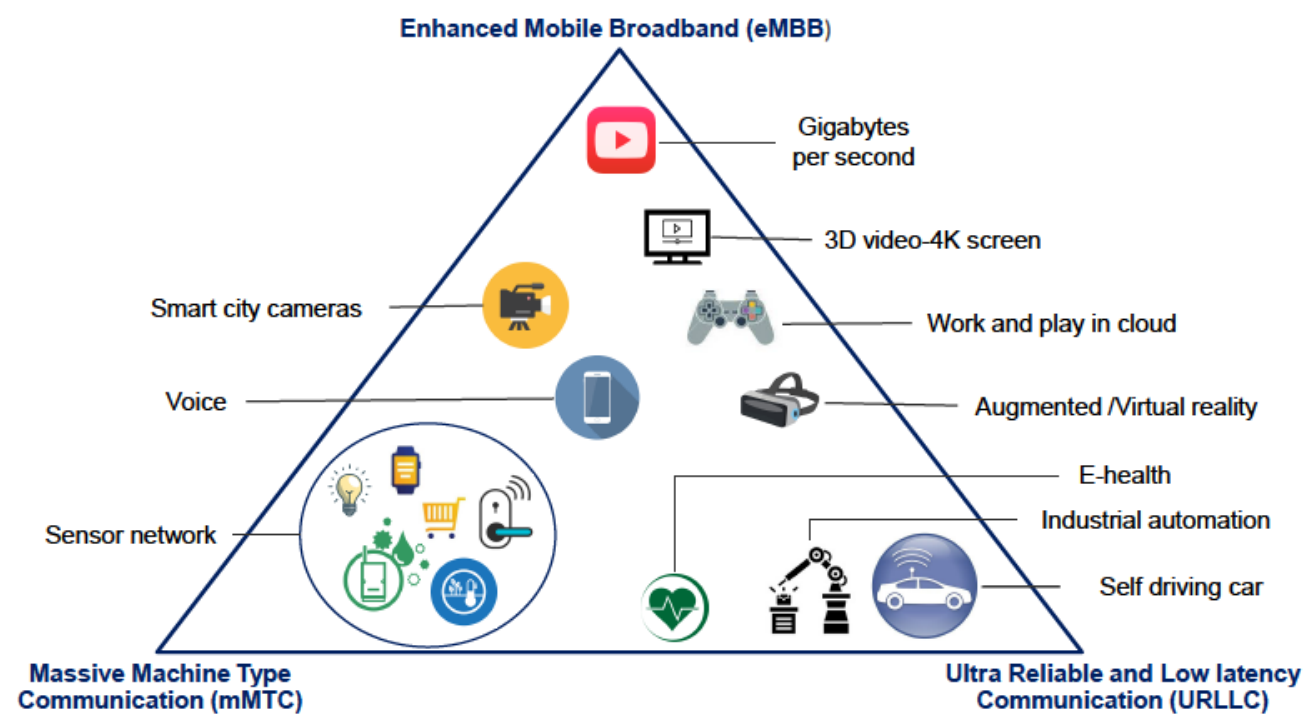

Figure 2.2: IoT applications under three service categories of 5G [11]

\section{URLLC}

Ultra-reliable and low latency communication refers to the short-packet communication services where data packets are exchanged at moderately low throughput but with strict requirements in low latency and high reliability. Latency in physical layer includes time for channel estimation, signal propagation from the transmitter to the receiver, encoding, decoding, re-transmission, scheduling, queuing and packet transmission [8]. Some examples of IoT application under this service category includes intelligent transport systems, vehicle-to-everything, transportation safety, smart grids, public protection and disaster relief, and wireless control of industrial manufacturing. The QoS requirement of this type of traffic is challenging to achieve since it has to satisfy ultra high reliability of packet error rate (PER) of $\left[10^{-9}-10^{-5}\right]$ and latency of $1 \mathrm{~ms}$ [10] [12]. These requirements come at the cost of increased resource use in the energy, frequency and spatial domains. 


\section{eMBB}

Enhanced mobile broadband service is an extended version of long term evolution (LTE) which is characterized by large payloads and aims at achieving 100-fold capacity increase over $4 \mathrm{G}$ systems. The QoS of this type of traffic includes high spectral efficiency, extreme data rates, and enhanced signal coverage per base station while guaranteeing a moderate reliability $[7,8,13]$. Examples of this type of applications include high-resolution video streaming, medical imaging, virtual reality, and augmented reality. In order to achieve such high performance, this service requires more robust physical layer technologies for example, high order modulation transmission, Massive MIMO transmission, cell densification and millimeter-Wave (mmWave) communication.

\section{mMTC}

Massive machine-type communication is a service category to support the access to a large number of devices which means providing wireless connectivity to tens of billions of low-complex low-power machine-type devices. Some of the features of mMTC involve small packet transmission, low data rate, sporadic user activity (for example, mixed traffic models with period and event-driven traffic), Uplinkdominated transmissions and battery constrained (low-energy) devices [14]. Example of mMTC-based IoT application includes sensing, tagging, metering, and monitoring. Contrary to eMBB and URLLC, the QoS of this type of application is high scalability (i.e, the complexity should scale linearly with the number of users, not exponentially), low power consumption and better energy efficiency. Also, the targeted reliability is lower than the other two services [7]. More details about the use cases of the three types of services and their QoS requirements can be found in [11]. 


\subsection{Background of Massive MIMO}

This section briefly reviews the technology of multiple-input multiple-output wireless communication, and then discusses the rationale behind MIMO evolution towards massive MIMO. Then it discusses the potential of Massive MIMO in accommodating the heterogeneity aspects of IoT.

\subsubsection{MIMO Review}

MIMO is an established wireless technology that has been incorporated into wireless broadband standards, such as IEEE 802.11n, IEEE 802.11ac, HSPA+, WiMAX and LTE. Fig. 2.3 shows examples of a cellular base station and a WiFi access point, both equipped with multiple antennas. Among these, IEEE 802.11ac and current LTE standard allows for up to eight antennas on base stations and on terminals [15].
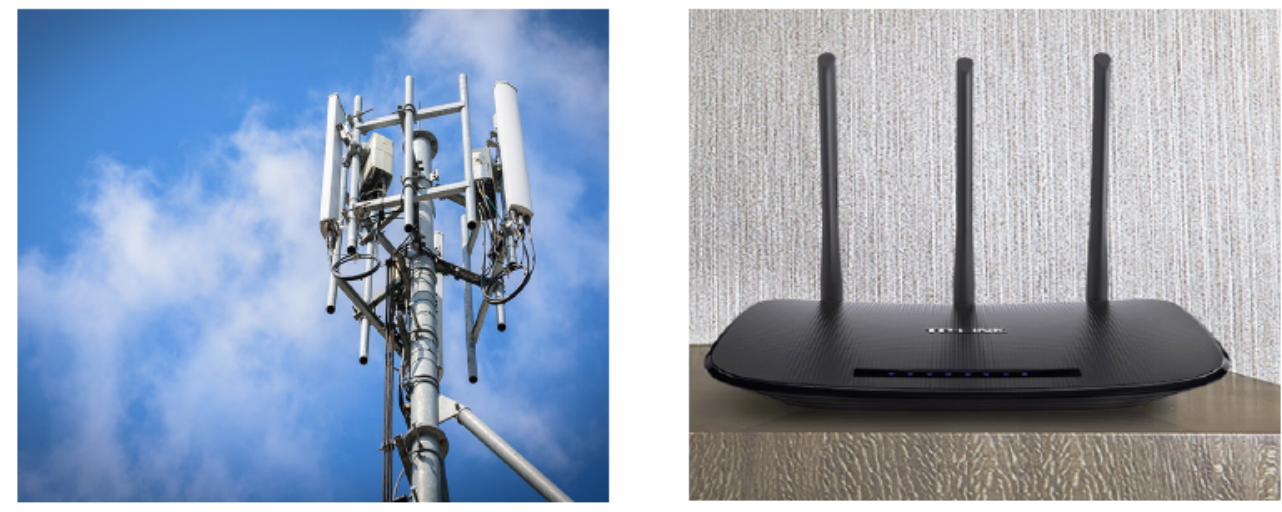

Figure 2.3: Examples of MIMO technology used in our everyday life. (Left) Tower with multiple antennas. (Right) TP-Link wireless router with multiple antennas (source: www.tp-link.com)

Generally, MIMO systems are divided into two categories: single-user MIMO (SUMIMO) and multi-user MIMO (MU-MIMO). Fig. 2.4 illustrates the two categories. In SU-MIMO, as the name suggests, multiple data streams are transmitted simultaneously to a single device using the same time/frequency resources. This results 
in increased coverage, peak throughput and reliability of communication with the help beamforming, spatial diversity and multiplexing technique. The challenge with SU-MIMO is that the user equipment becomes both hardware and computationally expensive because of several reasons. First, terminals need to have multiple antennas and thus independent electronics chains are required for each antenna. Secondly, terminals need to estimate the channel and perform decoding on downlink (DL) data. Therefore, it is not a suitable technology for many IoT devices.

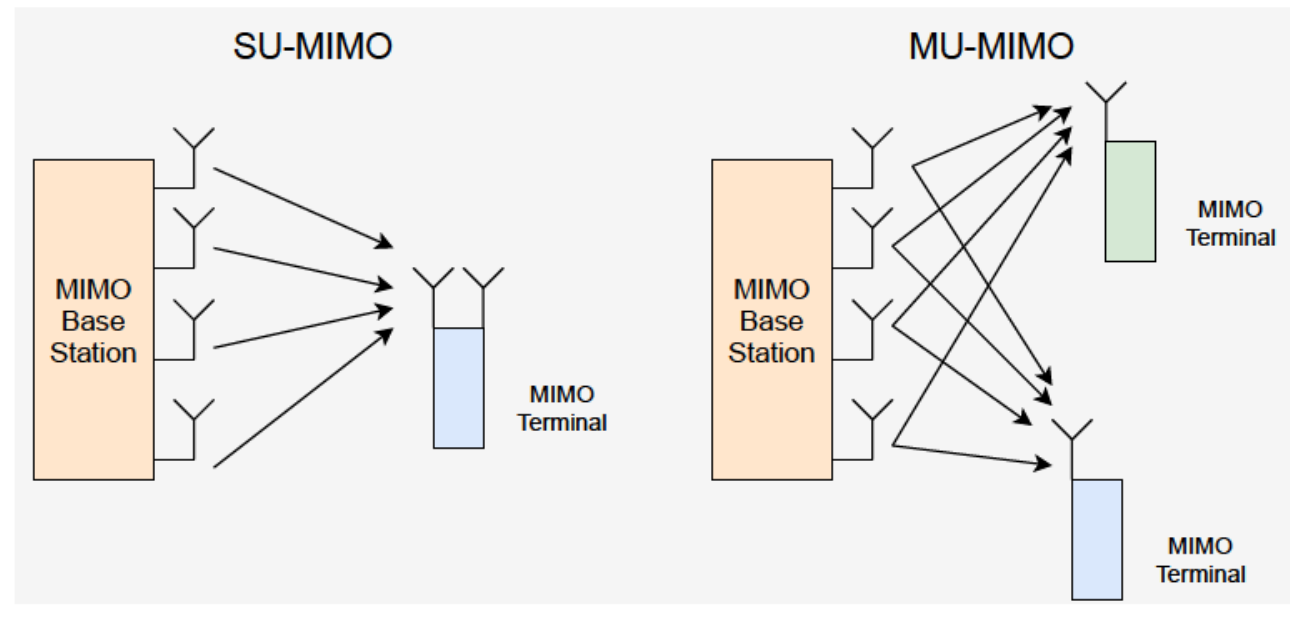

Figure 2.4: Single-user MIMO and multi-user MIMO.

The situation with MU-MIMO is radically different. Unlike SU-MIMO, MUMIMO allows to have single antenna devices provided that the typical angular separation between devices exceeds the angular resolution of the base station array [15-17]. Also, there should be no correlation between users either to transmit or receive data. The wireless channel is now spatially shared by different users, and the users transmit and receive without joint encoding and detection among them. All of the channel state information (CSI) determination and precoding of DL data is performed by the BS.. Therefore, user terminal in a MU-MIMO system has no additional complexity. The performance gains in terms of delay is attractive as it offers multiple access and 
broadcast capabilities and still achieve similar throughput gains as achieved in SUMIMO systems. However, the challenge of MU-MIMO is the requirement of spatial channel knowledge of all users for applying precoding technique at BS. Therefore, CSI becomes more crucial in MU-MIMO compared to SU-MIMO.

Although MU-MIMO offers improved performance over SU-MIMO, it does not provide scalability which is a crucial need for today's IoT driven wireless network. In next section, we will discuss the scalability issue faced by the existing SU-MIMO and MU-MIMO technology and how Massive MIMO evolved to address the challenge.

\subsubsection{Massive MIMO: A Scalable Technology}

In SU-MIMO, the fact that the prevents it from being scalable is the growing training burden for channel estimation. The main method for channel estimation is pilot signaling. In the DL, pilot symbols are transmitted from every antenna of the BS to the terminals. The terminals then estimate the channel responses (required for DPC) based on the received pilots signals, and feed them back to the BS to calculate precoding coefficients. In the UL, the terminals only need to send pilot signals to the BS so that it can decode it properly. Hence, scaling up the number of antennas requires more number of pilot symbols which increases the proportional amount of time to be spent in channel estimation [18].

In MU-MIMO, precoding and detection are designed for separating data streams with as little inter-user interference as possible. In the DL, the base station pre-filters signals to the intended users, and in the uplink (UL), the base station post-filters the received signals from all users. In conventional MU-MIMO, the BS needs to implement a complex signal processing scheme to achieve near-capacity performance. For example, applying a non linear precoding technique known as dirty paper coding (DPC) $[19,20]$ in the DL and detection schemes such as maximum-likelihood (ML) in 
the UL, can achieve optimal capacities. Unfortunately, the computational burden of this scheme grows exponentially with the size of the system (both number of antennas and terminals) and consume a large amount of circuit power, thereby becoming highly unsuitable for large scale operations.

In this regard, a breakthrough contribution was made by Marzetta in [21], where the concept of massive MIMO was proposed. As shown in Fig. 2.5, massive MIMO is an MU-MIMO technology where the BS is equipped with an excessively large number of $M$ antennas and it serves $K$ single- antenna terminals simultaneously on the same time frequency resource. The number of antennas needs to be greater than the number of active terminals i.e., $M>K$. Note that, with "antenna" we refer to a component with a size that is smaller than the wavelength and not the type of large high-gain antennas that are used at the BSs in conventional cellular networks. Deploying a large number of antennas at the BS results several interesting properties known as channel hardening, favourable propagation and array gain. In what follows, we briefly describe each properties of Massive MIMO that makes it a scalable and promising technology for future wireless communication. 


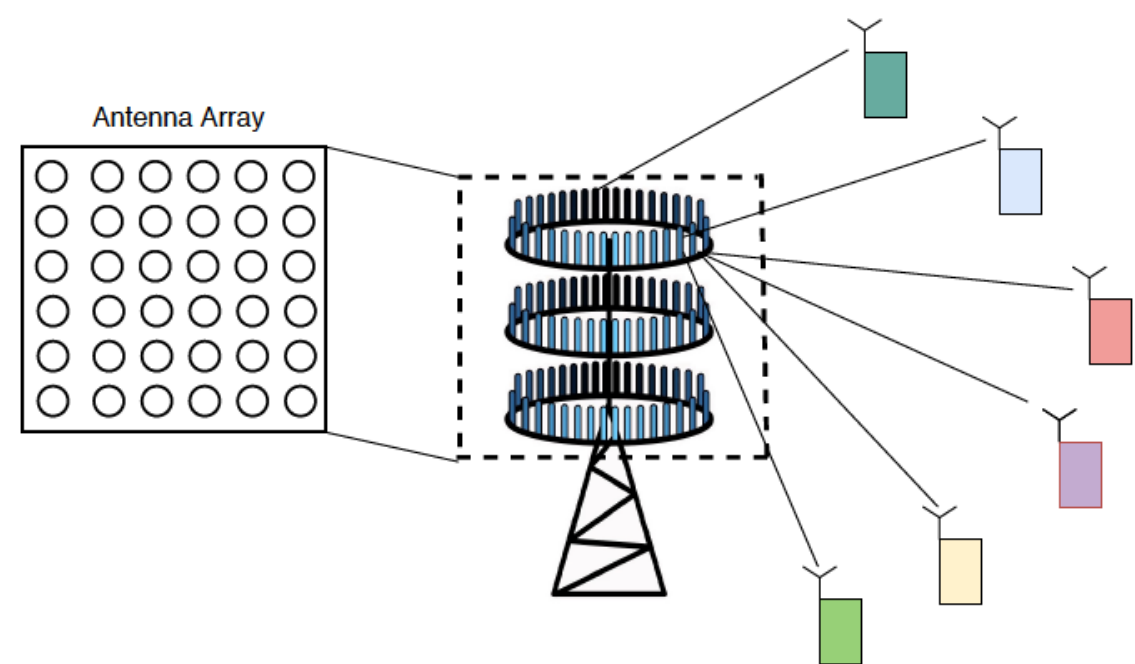

Figure 2.5: Massive MIMO : a multi-user MIMO technology where the BS exploits large antenna arrays to spatially multiplex data streams intended for different terminals.

\section{Channel hardening}

The term channel hardening was used in [22] to describe a fading channel that behaves as almost deterministic due to spatial diversity. The underlying principle of channel hardening is the strong law of large numbers. As more antennas are added, the normalized instantaneous channel gain converges to the deterministic average channel making the small-scale fading disappear [18,21,23]. This is attributed to the spatial diversity from having multiple receive antennas that observe independent fading realizations, which are unlikely to all be nearly zero simultaneously. Therefore, although the channel responses are random, its impact on communication is negligible.

\section{Favourable Propagation}

The favorable propagation concept provides a way to quantify the ability to separate UE (i.e, terminal) channels at BS with many antennas [24]. The interpretation is that 
the favorable propagation makes the directions of two terminal channels asymptotically orthogonal making the interference between each pair of UEs sufficiently small. When there are many active UEs, the total interference caused to a UE can indeed be large. Favorable propagation in this case makes it easier for the BS to mitigate interference between these UEs and allows us to use simple linear combining and precoding scheme to improve SE. Thus it alleviates the need for using complex non linear processing scheme such as DPC to improve the capacity. In fact, it is shown in [18] that by employing additional BS antennas, linear processing can achieve the same Shannon limit as achieved in DPC.

There are three prominent linear processing scheme in the current literature having similar operational meaning, namely matched filtering (MF), zero forcing (ZF) and minimum mean squared error (MMSE). The main difference lies in the precoding/decoding matrices which are function of the channel estimates and are given by

$$
\begin{aligned}
\mathbf{A}^{M F} & =\mathbf{H}, \\
\mathbf{A}^{Z F} & =\mathbf{H}\left(\mathbf{H}^{H} \mathbf{H}\right)^{-1}, \\
\mathbf{A}^{M M S E} & =\left(\mathbf{H H}^{H}+\frac{1}{\gamma} \mathbf{I}_{M}\right)^{-1} \mathbf{H},
\end{aligned}
$$

Here $\mathbf{A} \in \mathbb{C}^{M \times K}$ is the decoding/precoding matrix, $\mathbf{H}=\left[\mathbf{h}_{1}, \mathbf{h}_{2}, \ldots \mathbf{h}_{k}\right] \in \mathbb{C}^{M \times K}$ is the channel matrix and $\gamma$ is the average transmission signal to noise ratio (SNR). When the BS employs MF detection, it neglects the effects of inter-user interference and maximizes the received SNR. This detection technique requires minimal signal processing but experiences performance degradation in interference-limited scenarios because it neglects the effects of inter user interference in the system. On the other 
hand, when the BS employs ZF detection, it neglects the effects of noise and maximizes the received signal to interference ratio (SIR). Since this detection technique neglects the effects of noise in the system, it experiences performance degradation in noise-limited scenarios. For MMSE detection, the BS attempts to maximize the received signal to interference plus noise ratio (SINR). Since MMSE detection maximizes the received SINR, it performs better than MF and ZF detection techniques. However, it incurs higher computational complexity than the other two methods because it requires an increased number of matrix manipulations.

\section{Large Array Gain}

With more antennas, we obtain more the complex valued samples in the spatial domain, which means more degrees of freedom available for signal processing. If we coherently combine these samples, we can improve the SNR as compared to that in a single-antenna system [23]. This is because the BS can deploy multiple antennas to collect more energy from the electromagnetic (EM) waves which can be focused with extreme sharpness into small regions in space. This energy gain, achieved by using multi-antenna arrays, is known as array gain. Large array gain brings out great advantage for both UL and DL. In UL, the BS coherently combine the received signals from all antennas by leveraging the channel response of the desired terminals which in turn, improves the SNR. Therefore, without having the terminals increase their transmit power the $\mathrm{SE}$ and $\mathrm{EE}$ of the communication can be improved. In DL, the base station applies coherent superposition of wavefronts. It means that all wavefronts collectively emitted by all BS antennas add up constructively at the locations of the intended terminals, but destructively almost everywhere else. 


\subsection{Massive MIMO for IoT}

\subsubsection{System Model Description of Massive MIMO}

In this thesis, we are considering a single-cell massive MIMO where the system model is characterized as follows:

- The BS has $M \gg 1$ antennas to communicate with $K$ single antenna UEs with an antenna-UE ratio $M / K>1$. The location of the UEs follows uniform random distribution in the cell, excluding the disk centered on the base station. The terminals are generally physically separated by multiple wavelengths so that their channels are well modeled as statistically uncorrelated.

- The network uses spatial division multiple access (SDMA) to achieve a multiplexing gain by serving multiple nodes on the same time-frequency resources. A key element to performing wireless spatial multiplexing is an array of independently-controlled antennas. Each data signal is sent from all antennas but with different amplitude and phase to direct the signal spatially. In a cluttered propagation environment, the data streams can arrive from many directions simultaneously. The streams tend to reinforce each other constructively where desired, and interfere destructively where they are unwanted.

- We consider that the data is modulated using a practical discrete signal constellations scheme, such as Quadrature Amplitude Modulation (QAM). The network operates with synchronous TDD protocol to utilize channel reciprocity and limit CSI overhead. This assumption is important for massive MIMO because when we increase the number of antennas to improve network performance, it can incur complexity for the terminals. This is because if the number of the antennas increases at BS, each terminal would require to estimate more channel 
responses as the number of channel response is proportional to the number of BS antennas. As a result, the terminals will consume more power and there will be more CSI overhead and delay in the communication. Therefore, SDMA system is preferred to be combined with TDD for exploiting the reciprocity between UL and DL channels.

The operation of TDD protocol is illustrated in Fig. 2.6. The UL transmission is separated from the DL by the allocation of different time slots but they occur in the same frequency band. The physical propagation channels are reciprocal, thereby the channel responses are the same in both directions. The channel response is approximated as constant and flat fading over a coherence block. The coherence block consists of coherence bandwidth and coherence time over which the CSI is valid as shown in Fig. 2.6. If the coherence bandwidth is $B_{c}$ and coherence time is $T_{c}$, the coherence block contains $B_{c} T_{c}$ complex valued samples. To estimate the channel in the TDD mode, at first the UEs transmit mutually orthogonal pilot sequences to the BS on the UL. Then the BS acquire CSI based on the received pilot signals and use it for both UL receive combining and DL transmit precoding of payload data. As a result, operating in TDD protocol only requires pilots from the UEs, making the system independent of the number of antennas $(M)$. Also, there is no need for feedback channel. However, the challenge with TDD protocol is that the transmitter and receiver hardware of an antenna must be calibrated to maintain channel reciprocity.

- We assume that the base station perfectly knows the instantaneous channel matrix, and uses the knowledge for linear precoding and detection. CSI is only required at the base station to perform the data processing. 


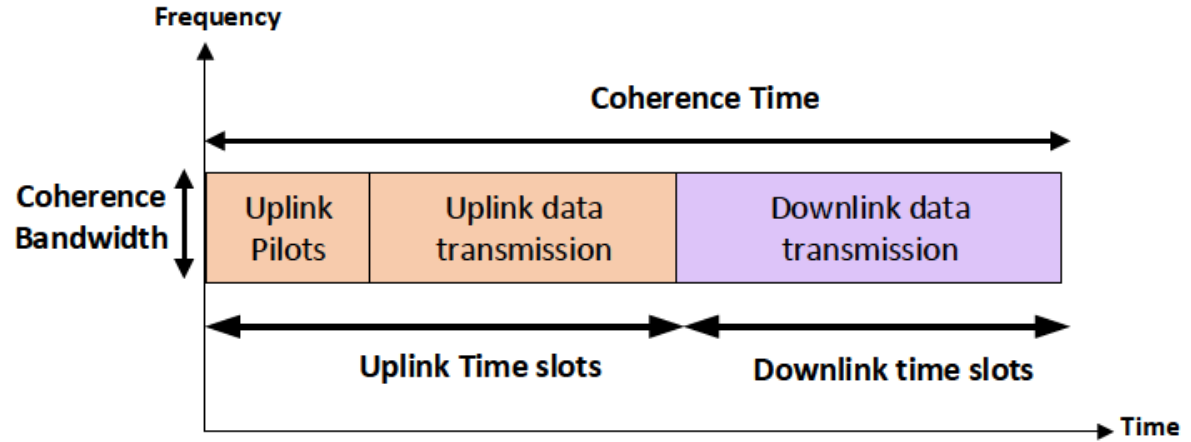

Figure 2.6: Illustration of TDD operation in a single coherence block.

Massive MIMO, of course, is not restricted to the above scenarios and assumptions.

Scenarios with, e.g., pilot contamination, inter-cellular interference, non-orthogonal wayeform design, and imperfect CSI, are all important aspects to investigate but are beyond the scope of this thesis.

\subsubsection{Uplink Transmission}

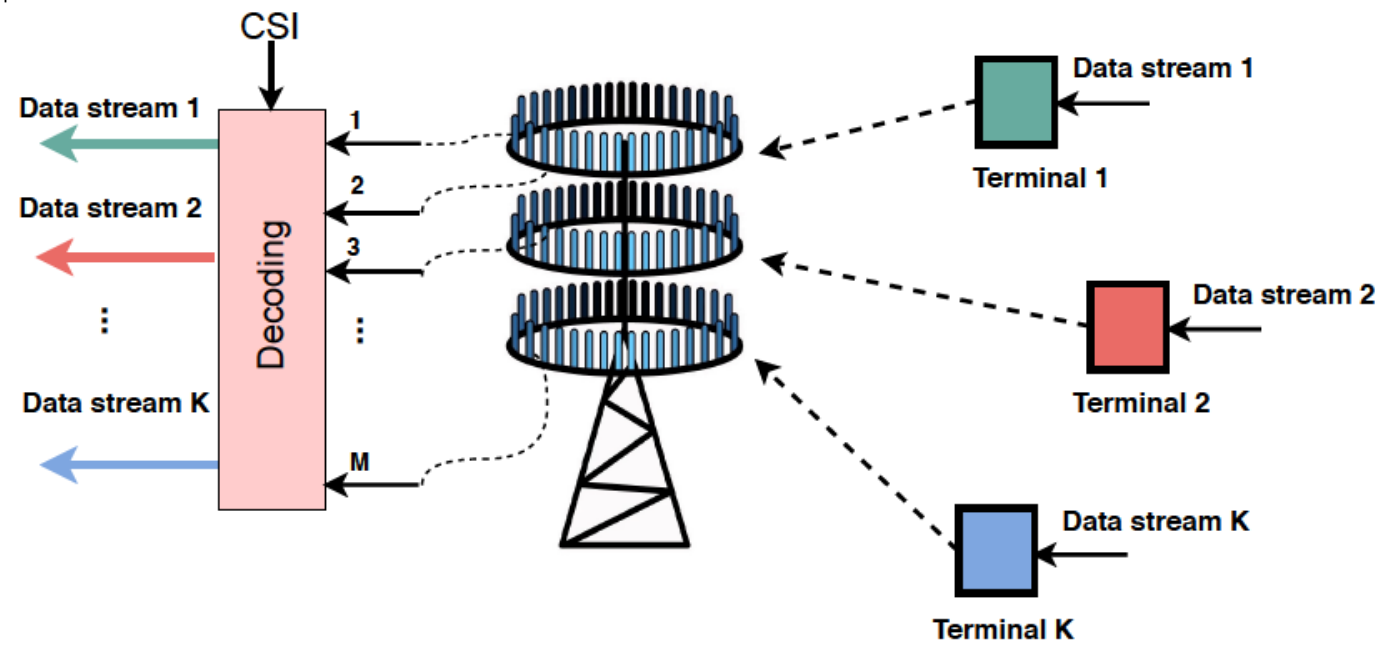

Figure 2.7: UL operation of Massive MIMO where $K$ number of terminals send data streams, all occupying the same time/frequency resources. The signals received by the antenna array are decoded at BS for separating the terminals. 
The UL operation of the Massive MIMO system is shown in Fig. 2.7. The user terminals transmit data streams bearing QAM symbols at the same time and over the same frequencies. As a result, the antenna array receives a superposition of the signals transmitted by its $K$ terminals. The wireless channel model is assumed to be uncorrelated Rayleigh fading or independent and identically distributed(i.i.d) Rayleigh fading. In the UL, the BS makes $M$ observations of the desired signal over its $M$ receive antennas. Let us consider the channel response from the $k$ th desired and interfering UEs is represented by $\mathbf{h}_{k} \in \mathbb{C}^{M}$ and $\mathbf{h}_{i} \in \mathbb{C}^{M}$ respectively. Here, $k=1,2, \ldots K$ and $i=1,2, \ldots K$. The $m$ th element of each vector is the channel response observed at the $m$ th $\mathrm{BS}$ antenna, for $m=1,2, \ldots M$. The channel response $\mathbf{h}_{k}$ and $\mathbf{h}_{i}$ is modeled as

$$
\mathbf{h}_{k}, \mathbf{h}_{i} \sim \mathcal{N}_{\mathbb{C}}\left(0_{M}, \beta \mathbf{I}_{M}\right)
$$

Here $\beta$ is the macroscopic large-scale fading, which includes distance-dependent pathloss, shadowing, antenna gains, and penetration losses while the Gaussian distribution accounts for small scale fading. The strength of the received desired signal is represented by average SNR. The average SNR of a UE is given by

$$
\mathrm{SNR}=\frac{p}{\sigma^{2}} \beta
$$

where $p$ denotes the UE's transmit power and $\sigma^{2}$ is the noise power. The information signals transmitted by the desired $k$ th UE and interfering UE is represented by $s_{k}^{u}, s_{i}^{u} \sim \mathcal{N}_{\mathbb{C}}(0, p)$ and the additive receiver noise is modeled as $\mathbf{n} \sim \mathcal{N}_{\mathbb{C}}\left(\mathbf{0}_{\mathbf{M}}, \sigma^{2} \mathbf{I}_{\mathbf{M}}\right)$. Therefore, the multi-antenna received UL signal at BS is given as

$$
\mathbf{y}_{o}=\sum_{k=1}^{K} \mathbf{h}_{k} s_{k}^{u}+\sum_{i=1, i \neq k}^{K} \mathbf{h}_{i} s_{i}^{u}+\mathbf{n}
$$


The BS then performs decoding by utilizing CSI, to separate the UEs in the spatial domain. We are considering simplest type of linear processing which is called MF processing. With this linear processing scheme, we use maximum ratio combining (MRC) for the uplink data transmission and maximum ratio transmission (MRT) for downlink data transmission. The purpose of the receive combing is to make the desired signal much stronger than the sum of interfering signals and noise. With MRC, the combining vector is $\mathbf{v}_{k}=\mathbf{h}_{k}$ that maximizes the relative power $\left\|\mathbf{v}_{k}^{H} \mathbf{h}_{k}\right\|^{2} /\left\|\mathbf{v}_{k}\right\|^{2}$ of the desired signal. The BS uses the knowledge of $k$ th UE's channel response to tailor a receive combining vector $\mathbf{v}_{k} \in \mathbb{C}^{M}$ to this UE channel. This vector is multiplied with the received signal in (2.6) to obtain

$$
\mathbf{v}_{k}^{H} \mathbf{y}_{o}=\sum_{k=1}^{K} \mathbf{v}_{k}^{H} \mathbf{h}_{k} s_{k}^{u}+\sum_{i=1, i \neq k}^{K} \mathbf{v}_{k}^{H} \mathbf{h}_{i} s_{i}^{u}+\mathbf{v}_{k}^{H} \mathbf{n}_{0}
$$

The achievable UL sum SE [bit/s/Hz] and a closed form lower bound using SDMA with MRC are given by [23] where the lower bound is obtained using Jensen's inequality

$$
\begin{aligned}
S E^{U L} & =\sum_{k=1}^{K} \mathbb{E}\left\{\log _{2}\left(1+\frac{p \mid\left\|\mathbf{h}_{k}\right\|^{2}}{\sum_{i=1, i \neq k}^{K} p \frac{\left|\left(\mathbf{h}_{k}\right)^{H} \mathbf{h}_{i}\right|^{2}}{\left\|\mathbf{h}_{k}\right\|^{2}}+\sigma^{2}}\right)\right\} \\
& \geq K \log _{2}\left(1+\frac{M-1}{(K-1)+\frac{1}{S N R}}\right)
\end{aligned}
$$

The factor $K$ in front of logarithm shows $\mathrm{K}$-fold gains in sum SE. This gain is called multiplexing gain of SDMA. This is achieved by serving UEs simultaneously and increasing the number of antennas to achieve an array gain. From eq. (2.8) we see that the sum SE increases proportionally to the number of UEs and the inside the logarithm, the desired signal power increases linearly with $M$. But as we increase 
$K$ the intra-cell interference power $(K-1)$ also increases linearly with $K$. The system can counteract the increasing interference by adding a proportional number of additional antennas. In other words, we need to maintain a antenna-UE ratio $(M / K)$ to maintain roughly same SINR per UE.

\subsubsection{Downlink Transmission}

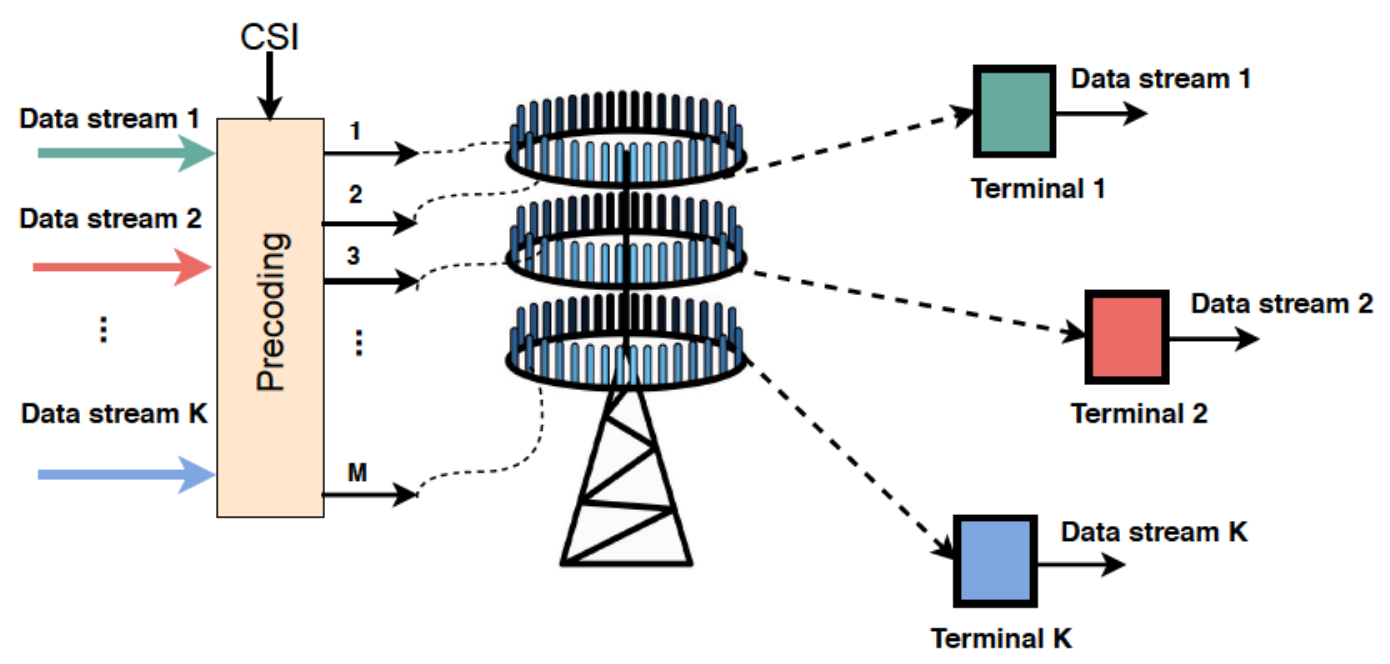

Figure 2.8: DL operation of Massive MIMO where the BS sends a separate signal to each the terminals from an array of $M$ antennas. The signals are precoded at BS such that it is received coherently at the terminal.

DL operation, as shown in the Fig. 2.8 is substantially the reverse operation of UL. Here the BS entails transmitting different data stream (containing QAM symbols) to each terminal using linear transmit precoding from an array of $M$ antennas. As a result, the $M$ transmit antennas have different channels to the receiving UE. The CSI of the UEs are utilized in the precoding block where the data streams are mapped into the signals that drive each of the antennas. Every signal sent from the antennas has different amplitude and phase that directs the signal spatially. This is known as beam-forming. By increasing the number of antennas, the beams can be focused more selectively to the terminals. Let us consider the DL channel response between 
the BS and $k$ th desired $\mathrm{UE}$ is $\mathbf{h}_{k}^{H}$. The receiver noise at this $\mathrm{UE}$ is denoted by $n_{k} \sim \mathcal{N}_{\mathbb{C}}\left(0, \sigma^{2}\right)$. The received DL signal $z_{k} \in \mathbb{C}$ at UE $k$ is modeled as

$$
z_{k}=\mathbf{h}_{k}^{H} \mathbf{w}_{k} s_{k}^{d}+\sum_{i=1, i \neq k}^{K} \mathbf{h}_{k}^{H} \mathbf{w}_{k} s_{i}^{d}+n_{k}
$$

where $s_{k}^{d}, s_{i}^{d} \sim \mathcal{N}_{\mathbb{C}}(0, p)$ is the signal transmitted to the $k$ th UE and $\mathbf{w}_{k} \in \mathbb{C}^{M}$ is the corresponding unit-norm precoding vector i.e, $\left\|\mathbf{w}_{k}\right\|^{2}=1$. It means that the total transmit power of BS is constant, irrespective of the number of antennas. With MRT precoding, we get the precoding vector as

$$
\mathbf{w}_{k}=\frac{\mathbf{h}_{k}}{\left\|\mathbf{h}_{k}\right\|} .
$$

Finally, the DL sum SE [bit/s/Hz] and closed form lower bound are given by [23]

$$
\begin{aligned}
S E^{D L} & =\sum_{k=1}^{K} \mathbb{E}\left\{\log _{2}\left(1+\frac{p\left\|\mathbf{h}_{k}\right\|^{2}}{\sum_{i=1, i \neq k}^{K} p \frac{\left|\mathbf{h}_{k}^{H} \mathbf{h}_{i}\right|^{2}}{\left\|\mathbf{h}_{i}\right\|^{2}}+\sigma^{2}}\right)\right\} \\
& \geq K \log _{2}\left(1+\frac{(M-1)}{(K-1) \frac{M-1}{M}+\frac{1}{S N R}}\right)
\end{aligned}
$$

Similar to UL, the multiplexing gain is also seen in eq. (2.11). The sum SE in DL and UL is very similar except for the multiplicative term $\frac{M-1}{M}$ in the denominator of (2.11) which is almost one for large $M$. We can make similar conclusion for DL that, to compensate the increased interference from the increasing $K$, more antennas need to be added to achieve an array gain. 


\subsubsection{Potential of Massive MIMO for IoT}

Massive MIMO can be a powerful technology in the quest of fulfilling the requirements of IoT connectivity. It is an enabler for future wireless infrastructure that will connect IoT devices with clouds and other network infrastructure. Some specific advantages of massive MIMO that play key role in IoT systems are:

- Energy Efficiency : The design of an energy efficient wireless system is one of the most important issues in IoT networks. With massive MIMO, the radiated EE can be significantly improved compared to current wireless systems. This is attributed to the large array gain, channel hardening and favourable propagation that stems from the large number of antennas at BS. It is shown in [25] that the transmit power of single antenna devices can be scaled down proportionally to the number of antennas at the BS with perfect CSI or to the square root of the number of BS antennas with imperfect CSI, to get the same performance as a corresponding single-input single-output (SISO) system.

- Coverage : Connectivity is the foundation of an IoT network. It is envisioned that billions of IoT devices will be connected in the near future which will require wide coverage on a massive scale. In fact, the connection density in some IoT applications can exceed thousands of devices $/ \mathrm{km}^{2}$ [10, 26, 27]. Considering the connection target, massive MIMO can increase the network capacity by several orders of magnitude without requiring additional spectrum. This is because this technology improves the sum SE of wireless networks by using spatial multiplexing to a large number of distributed UEs as mentioned in 2.3.2 and 2.3.3.

- Scalability : Wireless infrastructure in IoT must be scalable to support a growing amount of data demand from the increasing number of connected devices. 
In other words, the capability of the wireless system should have the potential to be enlarged through a proportional increase in resources without any degradation in the QoS. In this regard, massive MIMO is an excellent choice for IoT networks where the SE and EE of the system are directly related to the large number of antennas and UEs.

- Low complexity : Massive MIMO allows the spatial multiplexing gain at the base station to be obtained without the need for multiple antenna terminals, thereby allowing the development of small and cheap terminals while intelligence and cost is kept on the infrastructure side. The terminals do not even need to perform channel estimation. This physical layer flexibility for the terminal makes massive MIMO an ideal candidate for IoT system that can accommodate low power and low complex IoT device. From the BS perspective, the system is not only very robust because of spatial diversity obtained by using a higher number of antennas but also less complex because it allows the BS to use simple linear processing schemes.

\subsection{Related Work}

In this section, we provide a literature reviews for adaptive transmission in IoT application. At first, we provide related research work that focuses on improving SE and EE for single antenna IoT networks. Next, we provide the literature review of massive MIMO that motivated us to develop our traffic adaptive transmission scheme for massive MIMO based IoT networks. 


\subsubsection{Adaptive Transmission}

With the evolution of channel adaptive transmission technologies [28], many researchers aim at improving the SE and EE for IoT networks. To begin with, a statistical characterization for the energy consumption of adaptive transmission over fading channels has been presented in [29]. In [30] authors propose an adaptive modulation and power control scheme for energy efficient video transmission. Yunxia Chen et al. [31] designed a protocol using a greedy algorithm for minimizing energy consumption and maximizing the network lifetime that takes the channel fading characteristics and the data collection strategy of nodes into consideration. Adaptive modulation in a centralized cross-layered approach is investigated in [32] in order to minimize the total transmission energy of the network based on the different data rates that are required by the different sensors according to the application. Location aware adaptive modulation is studied in [33] for cluster based WSN to extend network lifetime by selecting the modulation scheme based on the location of nodes. In this context, nodes far away from the gateway use modulation scheme that will save their transmit energy. In [34], authors worked on finding optimal modulation mode considering latency and packet loss constraints in the wireless sensor network to minimize energy consumption.

An energy efficient data oriented transmission strategy for IoT is proposed in [35] where they consider QoS variation for long and short data transmission sessions over wireless fading channel. This authors also introduced new data-oriented performance limits and explored the outage performance of the data-oriented rate adaptive and power adaptive transmission schemes [36-38]. However, all of their works provide analysis for point to point communication and cannot give insight for a large number of devices.

In conclusion, adaptive transmission schemes for serving IoT nodes based on their 
remaining energy levels has not been considered in the existing literature. Hence, we propose a channel adaptive scheme in chapter 3 that takes the remaining energy level of the devices into consideration to allocate rate and power accordingly.

\subsubsection{Massive MIMO}

Over last few years, massive MIMO has gained significant attention in the literature. A comprehensive overview and survey on massive MIMO can be found in [39]. Massive MIMO in IoT application is well investigated in [1]. Standard EE-maximization techniques for millimeter wave based massive MIMO has been explored in [40]. Authors in [41] proposed an EE maximization problem for URLLC in massive MIMO that optimizes transmit power, bandwidth, and number of active antennas while taking the end to end delay and overall packet loss into consideration. In [42], the authors provided in depth analysis for energy efficient Massive MIMO systems to find the optimal number of antenna, terminals and power allocation. Their results showed that the number of antennas and terminals can be in the same order of magnitude to maintain EE. In [43] an adaptive antenna selection scheme in a multi-cell Massive MIMO scenario was proposed where the BS activates the number of antennas depending on the daily load variations. Authors in [44] have optimized the number of BS antennas and transmit power in the the DL of a Massive MIMO system under effective SINR constraint. Based on their result they conclude that the constraints on the transmission rate can be satisfied by optimizing the number of antennas. In [45] the authors have analysed the UL and DL performance in terms of achievable rate in multicell massive MIMO system taking imperfect channel estimation, pilot contamination, antenna correlation, and path loss into consideration. An OFDM based Massive MIMO system for improving energy efficiency of IoT application has been studied in [46] where the UL power control has been adopted for reducing devices power and peak-to-average 
power ratio reduction has been applied in DL transmission. A multi-objective resource optimization algorithm for massive MIMO is proposed in [47] that aims at maximizing network coverage and capacity while minimizing the total transmission power and inter-cell interference.

To conclude, a large area of research deals with applying massive MIMO for broadband communication that is applicable to particular IoT applications. On the other hand, using massive MIMO for machine type or ultra reliable communication is still a developing topic. Therefore, we propose a traffic adaptive transmission scheme that can optimally operate with different types of IoT traffic.

\subsection{Conclusion}

In this chapter, we provided background about IoT concepts along with the background of MIMO technology and how it evolved as Massive MIMO technology. Then we described the system model of massive MIMO and the achievable SE in UL and DL. After that, the potential of Massive MIMO for IoT systems was described. We then explained related work done in adaptive transmission and massive MIMO. In the next two chapters, we present our contributions using the above background to develop two adaptive transmission schemes for IoT. First one is for SISO based IoT system that deals with power heterogeneity of IoT devices and the second one is for massive MIMO based IoT network which considers IoT traffic heterogeneity. 


\section{Chapter 3}

\section{Adaptive Transmission for Heterogeneous IoT Sensor Nodes}

\subsection{Introduction}

With the recent trend of IoT, more and more devices are connected to the internet leading to a network of heterogeneous devices. IoT devices are therefore, diverse in terms of their power source and application requirement $[48,49]$. In addition to that, the connected nodes (i.e, terminals) exhibit different energy levels after some transmission period since the transmission usually occurs in sporadic fashion. Communicating with a large number of devices and sensors with diverse energy levels is not efficient with conventional channel-oriented transmission techniques since they are designed to utilize the channel quality to maximize throughout for any receiving node. But many low power IoT device operate with moderate rate and therefore, power saving will benefit them more as compared to achieving high throughput. Therefore, IoT transmission technique should take the energy level of the device into consideration to decide whether to utilize the channel quality for high throughput or to save energy at the cost of low but reasonable throughput. As such, it is important to develop a 
channel adaptive transmission scheme from the perspective of different energy levels of IoT node [50].

In this chapter, we propose a hybrid scheme (HS) for a SISO based IoT network which combines two transmission techniques, namely spectrally efficient scheme (SES) and power saving scheme (PSS). The proposed scheme incorporates a power management technique with the channel oriented strategy. It dynamically adapts the modulation mode and the transmit power according to the battery level (i.e, energy level) of the IoT nodes in each transmission session. More precisely, when a number of nodes transmit IoT data, the scheme first identifies whether the node is energy limited or not and then selects the transmission strategy based on its prevailing channel conditions.

The rest of this chapter is organized as follows. Section 3.2 introduces the system and channel models. Sections 3.3.1 and 3.3.2 discuss SES and PSS scheme, respectively, whereas the potential of the hybrid scheme is revealed in Section 3.3.3. Section 3.4 analyzes the performance of these schemes in terms of probability of data buffering, spectral efficiency, and power usage ratio (PUR). Section 3.5 confirms the derived analysis with simulations. Section 3.6 concludes the chapter with a brief summary.

\subsection{System and Channel Models}

\subsubsection{System model}

We consider the uplink communication of a IoT network where the sensor nodes are sending data to the BS as shown in Fig. 3.1. We consider a SISO model where the BS and the IoT sensor nodes are all equipped with a single antenna. We assume that the transceiver of the BS is fully adaptive and it has perfect received CSI from the sensor nodes. The number of IoT nodes are represented by $N_{s}$. Initially, every 
node has particular energy level denoted by $E_{r}$ which is different for each node and uniformly distributed in the range of $[0,1](0$ means an empty battery and 1 refers to a fully charged battery). The node that has battery level more than a predetermined threshold $E_{t_{h}}$ is considered to be a high power (HP) node, otherwise, it is classified as a low power (LP) node. Examples of a HP node is one that is directly powered by the grid or one that is battery power and has recently been charged to full power while a LP node example is one powered by battery whose battery level has dropped below a certain threshold. Nodes that are powered by renewable source will act as both LP and HP nodes depending on whether they are powered by renewable source or battery.

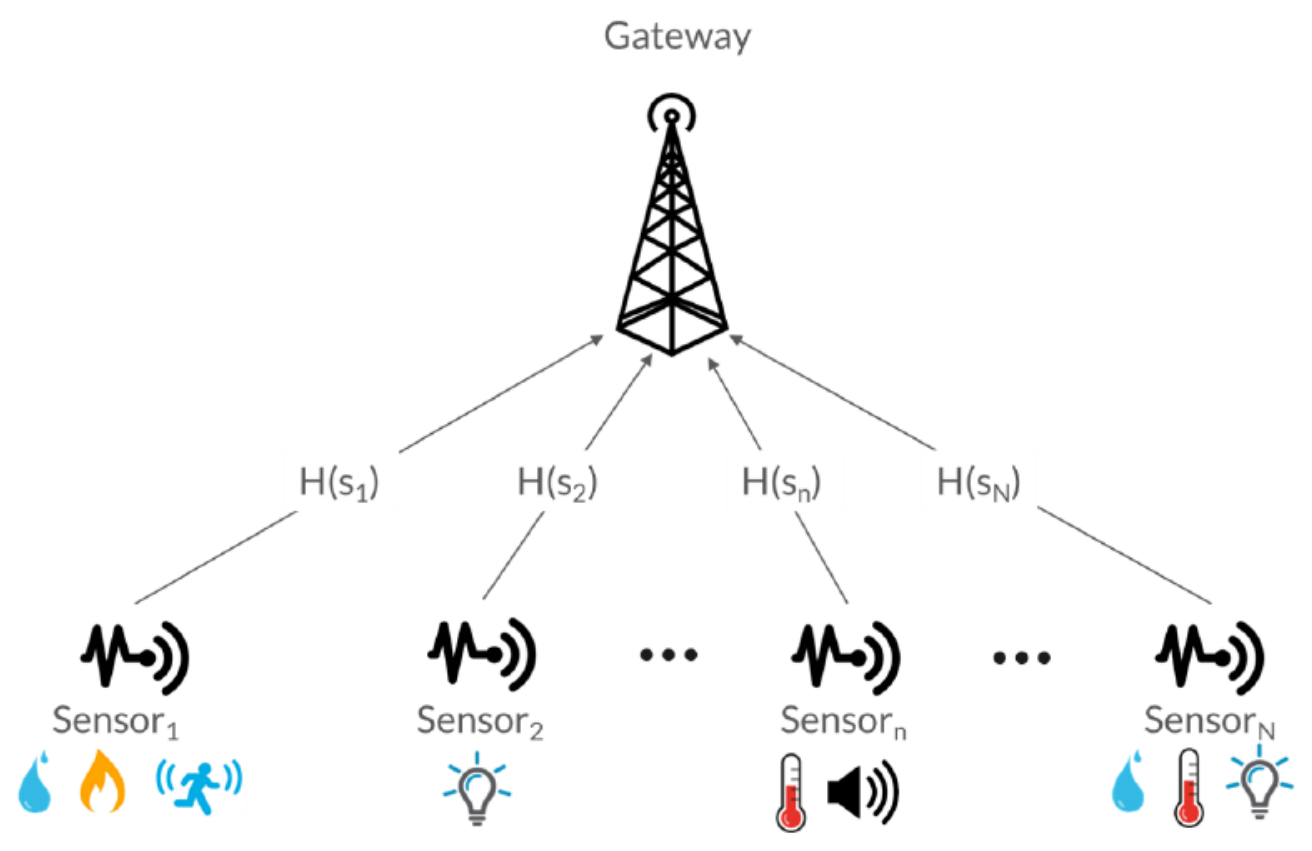

Figure 3.1: System Model

The BS will schedule the nodes by using max SNR scheduling that chooses the nodes with the best instantaneous received SNR in each transmission session. We assume the BS will get the information of the energy level of the scheduled node through the control channel. Based on the energy level information, it will determine 
the category of the scheduled node by comparing it to $E_{t_{h}}$. After that, it will choose the transmission scheme according to the proposed HS scheme.

\subsubsection{Channel Model}

The considered system operates in a time-slotted fashion through a block fading channel where the channel quality are time-varying but constant during one time-slot. The wireless channel experiences flat fading channel co-efficient $h$ with additive white Gaussian noise (AWGN). The instantaneous channel quality experienced by the nodes is characterized by the instantaneous received SNR defined as $\gamma=p_{t}\left|h^{2}\right| / \sigma^{2}$, where $p_{t}$ denotes the transmission power and $\sigma^{2}$ is the noise variance at the node. We assume that the received signal from each node experiences i.i.d Rayleigh fading. As such, the received SNRs from the $i$ th IoT sensor node, denoted by $\gamma_{i}$ for $\left(i=1, \ldots, N_{s}\right)$, follow an exponential distribution, with common probability density function (PDF) and cumulative distribution function $(\mathrm{CDF})$ given by

$$
f_{\gamma}(x)=\frac{1}{\bar{\gamma}} \exp \left(-\frac{x}{\bar{\gamma}}\right), \quad x \geq 0
$$

and

$$
F_{\gamma}(x)=1-\exp \left(-\frac{x}{\bar{\gamma}}\right), \quad x \geq 0
$$

respectively, where $\bar{\gamma}$ is the common average faded SNR.

In this chapter, we do not consider the effect of pathloss (i.e., large scala fading) and we only consider the Rayleigh fading (i.e., small scale fading). If we consider pathloss and we have fixed nodes, the node nearest to the gateway will have a very high probability of transmission which will be unfair to the other nodes. In order to address this, we can consider a round-robin transmission. While this new transmission scenario will provide fairness to all IoT nodes, it will come with a degradation in both 
the ASE and PUR (more on this in numerical results in section 3.5) as the results developed in this chapter are based on selecting the node with maximum received SNR. The analytical results presented in this chapter are more general than the case of round robin where we can easily generate the same performance metrics in a simpler and easier form. If the nodes are mobile and they have equal probabilities of being near the base station, then the fairness issue is not applicable even when selecting the node with the highest SNR. In the following chapter, in a more practical framework, we consider both small and large scale fading scenarios when proposing the traffic adaptive transmission technique.

\subsection{Modes of Operation}

\subsubsection{Spectral Efficient Scheme (SES)}

We adopted adaptive modulation (AM) as a spectral efficient scheme for IoT application. AM is a practical rate adaptive transmission scheme that can adjust modulation scheme with instantaneous channel condition, while maintaining an acceptable bit error rate (BER) performance. The performance of this scheme is based on the channel gain that means, the more the channel gain, the higher transmission rate is supported which results in less transmission delay. Therefore, AM is a suitable candidate for spectrum efficient, reliable and fast transmission of IoT data over fading wireless channels. In this context, we are using M-ary QAM as the adaptive modulation system. To perform the AM operation, the value of received SNR is divided into $N+1$ fading regions and the constellation size $M=2^{n}$, where $n$ is the number of bits per symbol, is assigned to the $n$th region $(n=0,1, \ldots N)$ The boundary SNR $\gamma_{n}$ are typically determined such that the instantaneous BER for selected modulation scheme is below a target BER value [51]. The BER of $2^{n}$-QAM constellations with $\mathrm{SNR}$ of $\gamma$ is 
approximated as [52] by

$$
P_{b_{n}}(\gamma) \simeq \frac{1}{5} \exp \left(\frac{-3 \gamma}{2\left(2^{n}-1\right)}\right)
$$

Given a target instantaneous BER equal to $P_{b_{0}}$, the adaptive modulator switching thresholds are given by

$$
\gamma_{T_{n}}=-\frac{2}{3} \ln \left(5 P_{b_{0}}\right)\left(2^{n}-1\right) ; n=1,2, \ldots, N
$$

If the maximum instantaneous SNR $\gamma_{m}$ falls into the $n$th region, the receiver feeds back the index of the chosen modulation to the selected node via an error-free feedback channel. After that, the node and the receiver communicate with modulation mode $n$ using $2^{n}$-QAM. The transmit power to operate with $\gamma_{m}$ is represented by $P_{t_{m}}$. If $\gamma_{m}$ of all the nodes are less than threshold for the lowest modulation mode $\gamma_{T_{1}}$, then no node will be scheduled for transmission. As a result, no node can transmit and the data will be buffered during that slot.

In the AM scheme, good channel gain is translated in higher modulation mode that results in high throughput at the cost of high transmit power. Therefore, this scheme is not suitable for power limited sensors. To this end, the PSS can be used to minimise the power consumption for IoT sensor nodes.

\subsubsection{Power Saving Scheme (PSS)}

We are using continuous power adaptation as power saving scheme which allows the IoT nodes to adapt their transmit power according to the fading channel conditions while satisfying the BER constraint. When $i$ th node experiences a received SNR $\gamma_{i}$, which falls into $n$th fading region, the receiver will ask the node to reduce its power to 
$\gamma_{T_{1}}$. This will result in $\mathrm{dB}$ SNR saving of $\left(\gamma_{i}-\gamma_{T_{1}}\right)$ for the node. The transmit power to operate with lowest modulation mode $\gamma_{T_{1}}$ is denoted by $P_{t_{1}}$. Higher channel gain will result in higher power saving because the scheme uses a constant modulation mode with lowest constellation size. However, this scheme does not provide high throughput and may incur delay while transmitting large amount of data.

\subsubsection{Hybrid Scheme (HS)}

To offer a tradeoff between the SES and PSS schemes, we develop a hybrid algorithm to utilize the advantages of each scheme and distribute the resources according to IoT nodes' capability. When the AM or power adaptation schemes are implemented as wireless transmission scheme, the energy level diversity among the nodes becomes more significant since the schemes are based on fading channel gain. Therefore, we design the hybrid scheme that combines the channel oriented scheme with the energy level $E_{r}$ of the node to facilitate the HP and LP node. The mode of operation of the proposed hybrid scheme is summarized in the flowchart presented in Fig. 3.2. 


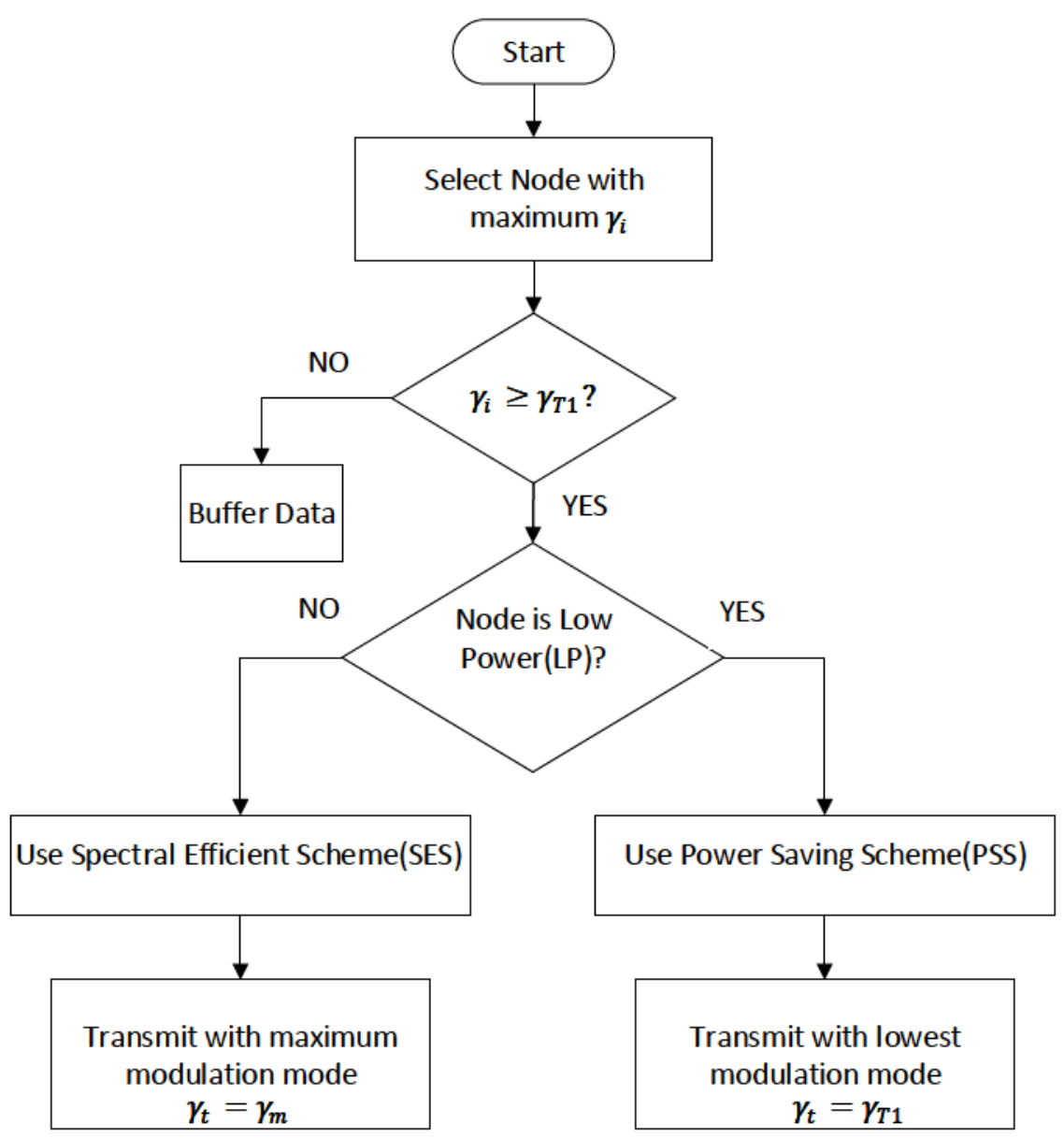

Figure 3.2: Hybrid scheme's mode of operation

In the beginning of every time slot, the node with maximum SNR $\gamma_{m}$ is selected by the receiver and then compared with the threshold SNR $\gamma_{T_{1}}$. If the minimum modulation mode cannot be met, then data is buffered for that time slot. Otherwise, the receiver identifies the node type by comparing $E_{r}$ with $E_{t h}$. If the node type is identified as LP, the receiver uses PSS scheme for communication and the selected node transmits with reduced power $P_{t_{1}}$. This allows the node to save power and stay longer in the network. On the other hand, if the node is a HP node, the receiver will use the SES scheme as a mode of transmission that will transmit with its maximum 
available power $P_{t_{m}}$ and rate supported by the channel gain. The value of the threshold $E_{t h}$ plays an important role in this scheme. If the system sets a higher $E_{t h}$ value, more nodes will be classified as LP node and vice versa. In following sections, we show how the energy level of the nodes and the threshold value affects the systems resource utilization.

\subsection{Performance Evaluation}

\subsubsection{Delay Performance}

In this chapter, we consider an IoT system that buffers data anytime the channel is not reliable. In this context, if the maximum received SNR from all IoT sensor nodes is below the threshold for the minimum constellation size, i.e. $\gamma_{T_{1}}$, then no transmission takes place during that time slot. Therefore, the delay for all schemes is the same and is measured in terms of the probability of data buffering, $P_{\text {buf }}$. This probability is given by

$$
\begin{aligned}
P_{\text {buf }} & =\operatorname{Pr}\left[\gamma_{m}=\max _{i=1: N_{s}} \gamma_{i}<\gamma_{T_{1}}\right] \\
& =\prod_{i=1}^{N_{s}} \operatorname{Pr}\left[\gamma_{i}<\gamma_{T_{1}}\right] \\
& =\left[F_{\gamma}\left(\gamma_{T_{1}}\right)\right]^{N_{s}} \\
& =\left[1-\exp \left(-\frac{\gamma_{T_{1}}}{\bar{\gamma}}\right)\right]^{N_{s}}
\end{aligned}
$$

\subsubsection{Average Spectral Efficiency}

The average spectral efficiency is measured in terms of average number of transmitted bits per channel use (bpcu). As explained in the mode of operation of the proposed schemes, the PSS schemes aims at minimizing the energy usage while transmitting 
with one bpcu when the maximum received SNR is above $\gamma_{T_{1}}$ (otherwise, data is buffered as explained in subsection 3.4.1). On the hand, the ASE for the SES and the HS depends on the average SNR and is given in what follows.

\section{SES}

A general expression of the average spectral efficiency of an adaptive modulation system is given in [52, Eq. (33)] by

$$
\eta=\sum_{n=1}^{N} n p_{n}
$$

where $p_{n}$ denotes the probability that the $n$th constellation is used. The expression of this probability is given for the SES scheme by

$$
p_{n}=F_{\gamma_{m}}\left(\gamma_{T_{n+1}}\right)-F_{\gamma_{m}}\left(\gamma_{T_{n}}\right)
$$

where $\gamma_{m}$ is the maximum received SNR from all IoT sensor nodes.

Using the above expression of $p_{n}$ and (3.6) we obtain the following expression of the average spectral efficiency of the SES scheme

$$
\eta^{\mathrm{SES}}=N-\sum_{n=1}^{N}\left[1-\exp \left(-\frac{\gamma_{T_{n}}}{\bar{\gamma}}\right)\right]^{N_{s}} .
$$

HS

Depending on the type of the IoT sensor node and on the channel quality, the ASE for the hybrid scheme features three different scenarios: (i) if the minimum modulation mode cannot be satisfied then data is buffered and the ASE is then equal to zero, and if the minimum modulation mode is met then there the two remaining scenarios occur based on the device type (ii) LP device will use BPSK while reducing the transmit 
power as described in PSS scheme, otherwise, (iii) HP device will transmit with the highest possible constellation size using SES scheme. Therefore, the ASE for the HS is given by

$$
\begin{aligned}
\eta^{\mathrm{HS}} & =\operatorname{Pr}\left[E_{r}<E_{\mathrm{th}}\right] \operatorname{Pr}\left[\gamma_{m} \geq \gamma_{T_{1}}\right]+\operatorname{Pr}\left[E_{r} \geq E_{\mathrm{th}}\right] \eta^{\mathrm{SES}} \\
& =E_{\mathrm{th}}\left(1-\left[1-\exp \left(-\frac{\gamma_{T_{n}}}{\bar{\gamma}}\right)\right]^{N_{s}}\right) \\
& +\left(1-E_{\mathrm{th}}\right)\left(N-\sum_{n=1}^{N}\left[1-\exp \left(-\frac{\gamma_{T_{n}}}{\bar{\gamma}}\right)\right]^{N_{s}}\right)
\end{aligned}
$$

\subsubsection{Power Usage Ratio}

The power usage ratio by an IoT sensor node is equal to the ratio between the transmit power $P_{t}$ and the maximum transmit power $P_{t_{m}}$. As explained in the mode of operation of the schemes, the SES scheme aims at maximizing the ASE while transmitting with the maximum possible power and therefore, does not have any power saving. In this case, the IoT node will use $P_{t}=P_{t_{m}}$ for transmission and the PUR is equal to one. We therefore, skip the PUR calculation of SES scheme for brevity. On the other hand, the PSS is designed to save power by using less average transmit power which results in higher PUR. HS scheme combines the two schemes to facilitate the HP and LP nodes in the network. In what follows, the PUR for PSS and HS schemes are provided. 


\section{PSS}

The power usage recorded by a sensor node when a transmission takes place is given by $\gamma_{T_{1}} / \gamma_{m}$. Therefore, the PUR for the PSS is given by

$$
\begin{aligned}
& \mathrm{PUR}^{\mathrm{PSS}}=\mathbb{E}\left[\gamma_{T_{1}} / \gamma_{m}\right] \\
& =N_{s} \gamma_{T_{1}} \int_{0}^{\frac{1}{\gamma_{T_{1}}}} \frac{\exp \left(-\frac{1}{\bar{\gamma} x}\right)}{\bar{\gamma} x}\left[1-\exp \left(-\frac{1}{\bar{\gamma} x}\right)\right]^{N_{s}-1} d x
\end{aligned}
$$

\section{HS}

The power saving recorded by a sensor node for the hybrid scheme depends on the channel condition and the device's remaining power. Following the same three scenarios discussed when evaluating the ASE, the PUR for the hybrid scheme is given by

$$
\begin{aligned}
\mathrm{PUR}^{\mathrm{HS}} & =\operatorname{Pr}\left[E_{r}<E_{\mathrm{th}}\right] \mathrm{PUR}^{\mathrm{PSS}} \\
& +\operatorname{Pr}\left[E_{r} \geq E_{\mathrm{th}}\right] \operatorname{Pr}\left[\gamma_{m} \geq \gamma_{T_{1}}\right] \\
& =E_{\mathrm{th}} \mathrm{PUR}^{\mathrm{PSS}} \\
& +\left(1-E_{\mathrm{th}}\right)\left(1-\left[1-\exp \left(-\frac{\gamma_{T_{n}}}{\bar{\gamma}}\right)\right]^{N_{s}}\right)
\end{aligned}
$$

\subsection{Numerical Results}

In this section, we evaluate the performance of the proposed schemes as a function of an average SNR $\bar{\gamma}$ ranging from $0 \mathrm{~dB}$ to $20 \mathrm{~dB}$ while considering Rayleigh fading. Taking, for example, an Average BER of $10^{-3}$, the adaptive modulation switching 
thresholds are given by

$$
\gamma_{n}=\left\{\begin{array}{c}
\gamma_{1}=3.53 \\
\gamma_{2}=10.60 \\
\gamma_{3}=24.73 \\
\gamma_{4}=52.98
\end{array}\right.
$$

\subsubsection{Data Buffering}

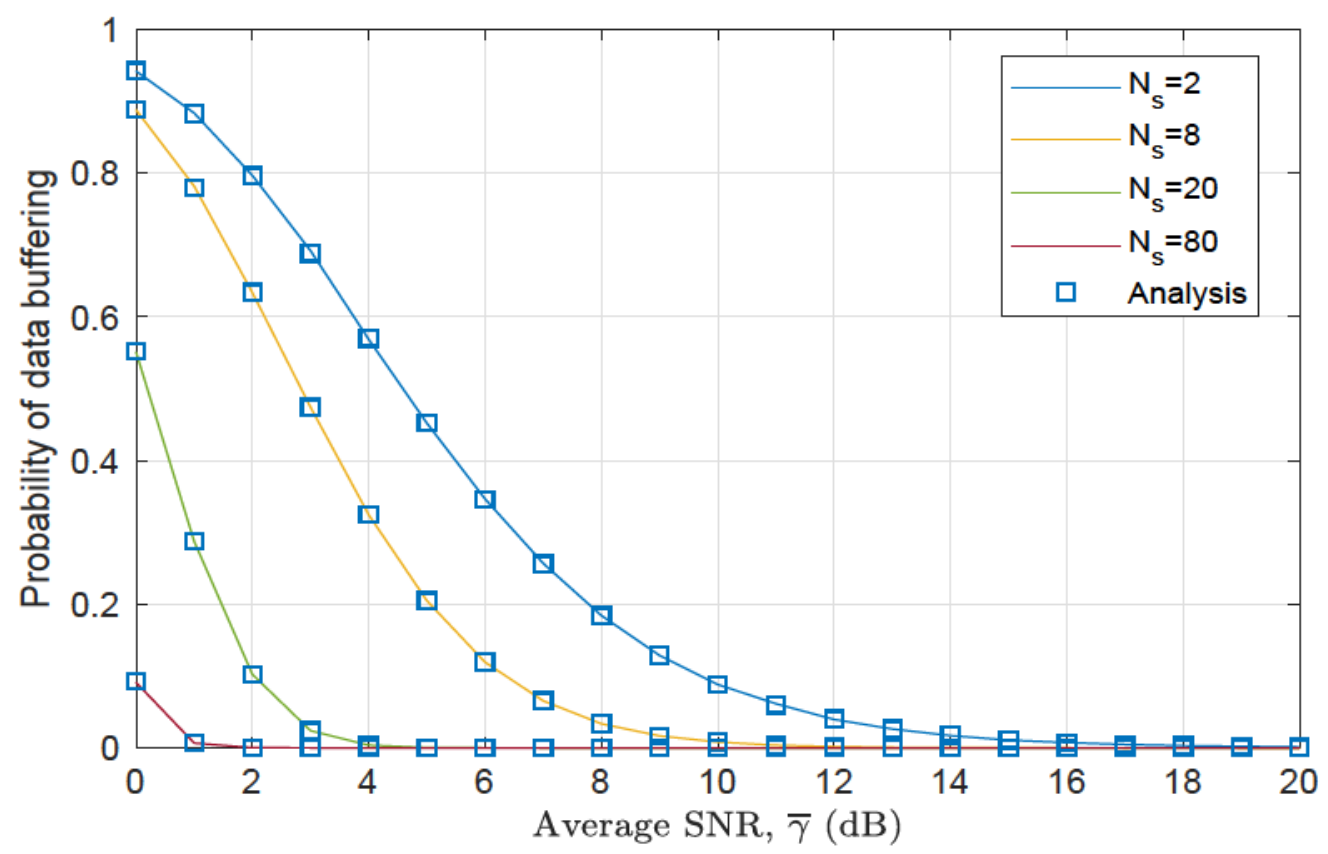

Figure 3.3: Probability of data buffering

Fig. 3.3 depicts the probability of data buffering as a function of average SNR. This performance is basically the outcome of max SNR scheduling considered in the system model. As expected, delay is reduced with higher $\bar{\gamma}$ since $\gamma_{T_{1}}$ threshold is verified. This Fig. also confirms that the increment of nodes significantly reduces the probability of data buffering. This is because higher number of node $N_{s}$ will lead to 
higher probability that at least one node will be experiencing $\gamma_{T_{1}}$.

\subsubsection{Power Usage Ratio}

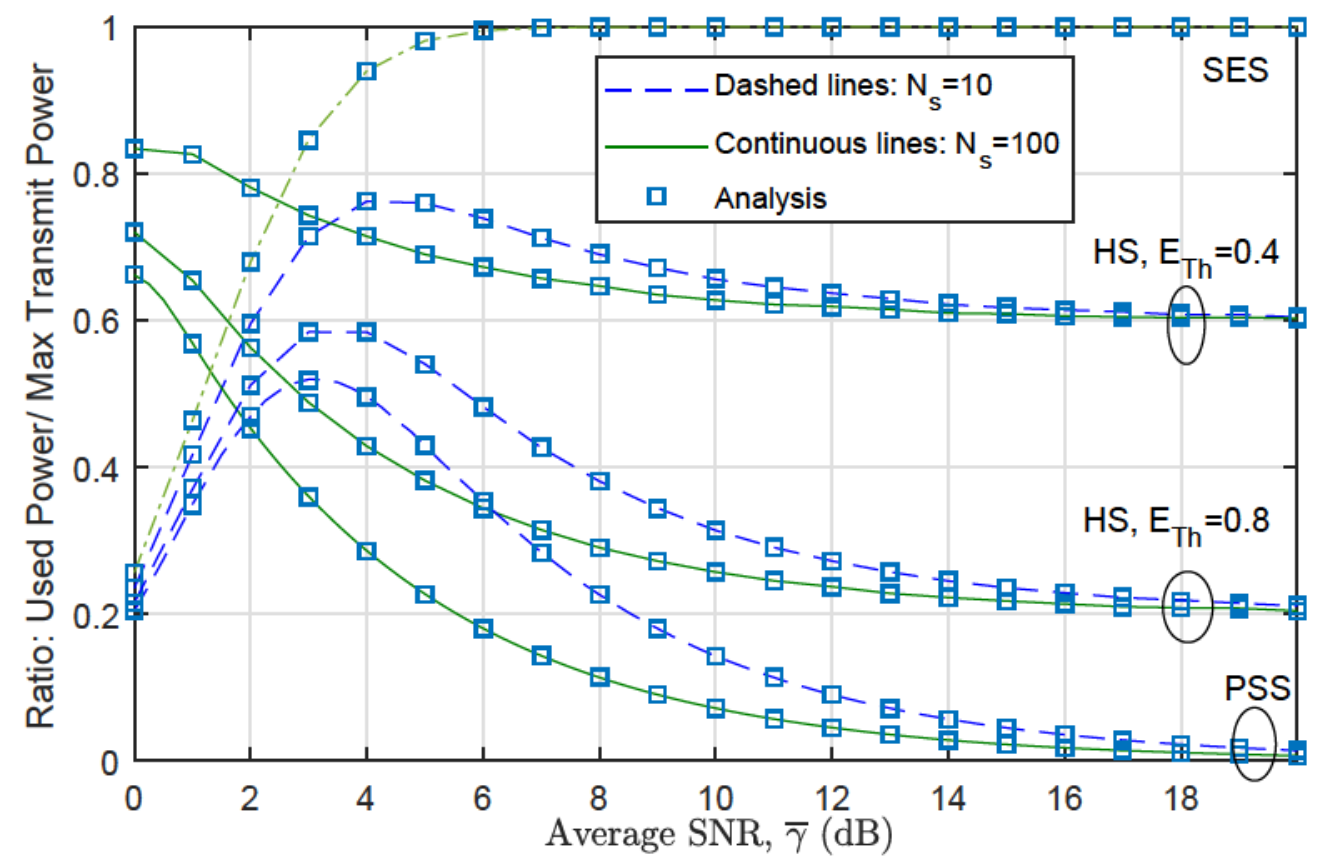

Figure 3.4: Average Power Consumption: Ratio of power used by the maximum transmit power

Fig. 3.4 compares the average transmit power usage ratio of the three schemes using small and large number of nodes. As shown in Fig. 3.4, the SES scheme starts with approximately $20 \%$ of power usage then it goes up to $100 \%$ with the increment of average SNR. 100\% power usage is expected for the SES scheme since it uses the maximum available transmit power. On the other hand, PSS shows two different scenarios for small and large number of nodes under low average SNR. For $N_{s}=10$, the PUR rises because the difference between the used power and maximum power is not significant. But as average SNR increases the allowable maximum transmit power increases and more power saving is achieved. For $N_{s}=100$ the nodes start to 
use $65 \%$ of their available power due to a lower probability of data buffering and then reduces to almost $5 \%$ of the power usage. High average SNRs result in more power saving for the PSS scheme that results in lower PUR.

The trend of PUR for hybrid scheme is in the middle of two other schemes because of the combined effect from HP and LP nodes. Unlike SES, it will not use maximum power for all nodes but it will give that opportunity only to the HP node that has remaining battery level $E_{r}$, more than $E_{T_{h}}$. The larger value of $E_{T_{h}}$ results in less number of HP node and more number of LP node. Since LP node follows the PSS scheme, higher $E_{T_{h}}$ implies in more power saving and less PUR. From the result in Fig. 3.4 we can conclude that the ASE of HS scheme depends on the number of LP and HP nodes in the system which is defined by the value of $E_{T_{h}}$. Therefore, when $E_{T_{h}}$ approaches to 1 or 0 , the system behaves like homogeneous network consist of only LP nodes or HP nodes respectively.

\subsubsection{Average Spectral Efficiency}

Fig. 3.5 shows the ASE as a function of the average SNR $\bar{\gamma}$ using different values of $E_{T_{h}}$ for two different number of nodes. In all cases of $N_{s}$, SES shows the maximum SE whereas the PSS shows the minimum. This is again because the SES operates with $\gamma_{m}$ to transmit maximum bpcu but PSS applies $\gamma_{T_{1}}$ to transmit only $1 \mathrm{bpcu}$ under all channel conditions. On the other hand, HS considers the diversity of energy level among IoT nodes and works on classifying them based on $E_{T_{h}}$. Since small value of $E_{T_{h}}$ implies the probability of having more HP node in the system that can use SES and therefore, the average ASE of the network increases. Similarly, high $E_{T_{h}}$ classifies most of the node as LP node that can not follow SES which results in low average spectral efficiency. We can conclude that the HS scheme can balance the trade-off between power and SE and therefore, a suitable resource management technique for 
IoT network.

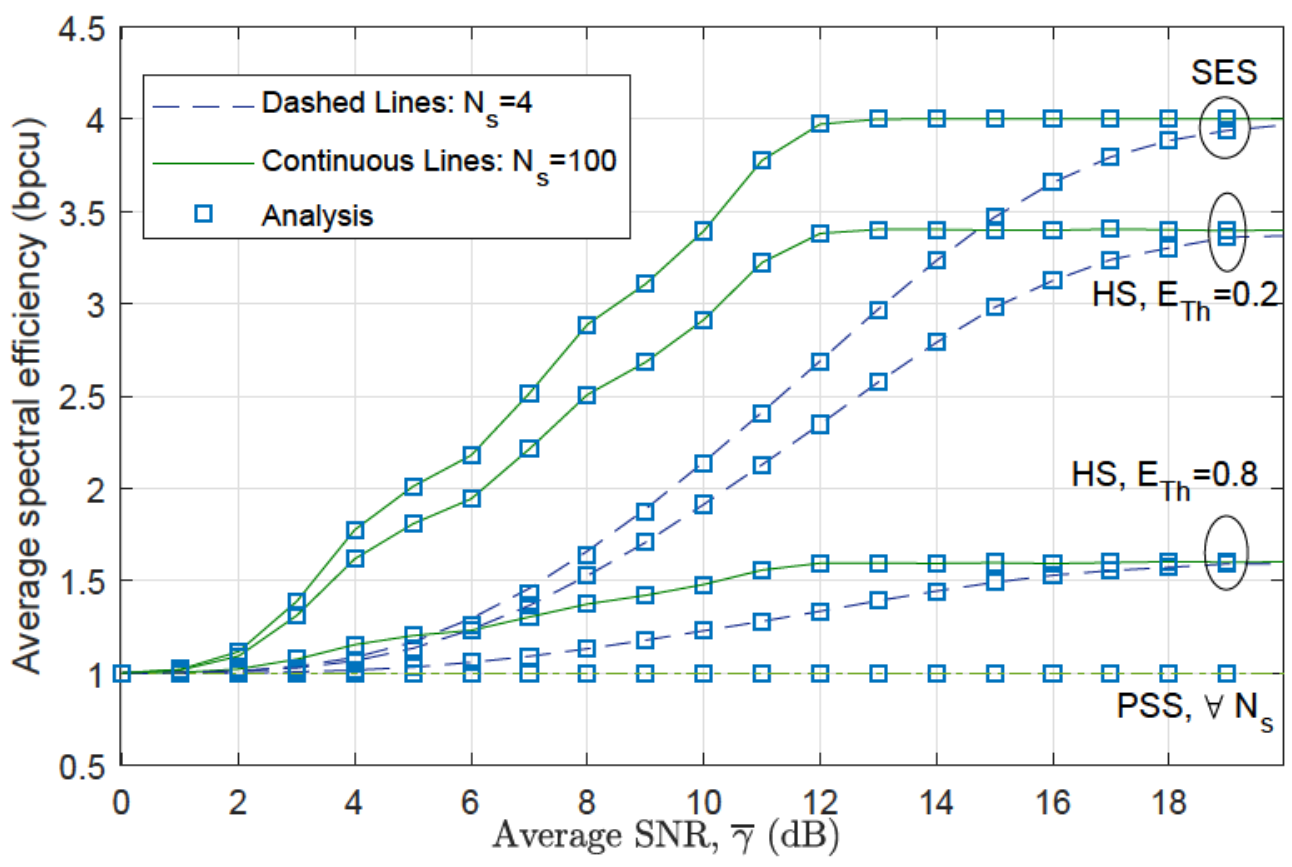

Figure 3.5: Average spectral efficiency

\subsection{Conclusion}

In this chapter, we have used a simplified IoT network that communicates according to single antenna single user system. Using adaptive modulation and power adaptation, the hybrid scheme is proposed as a trade-off between SE and EE while taking the remaining energy level of IoT node into consideration. The scheme was shown to take advantage of high data rate achieved by AM and high power saving gained from power adaptation. This will allow the low power node to sustain more time in the network and high power node to improve the performance of data transmission. Simulation results show that HS scheme is able to manage the power based on the different energy levels of the nodes. In our next chapter, we consider more robust IoT network that operates with massive MIMO technology and deals with different IoT traffic. 


\section{Chapter 4}

\section{Traffic Adaptive Transmission Schemes for Massive MIMO based IoT network}

\subsection{Introduction}

Energy efficiency and massive connectivity is becoming increasingly essential for IoT. The BS is expected to serve large number of IoT devices in an energy efficient way but unlike traditional broadband service, IoT connectivity has requirements and constraints that are significantly diverse $[1,8,12,13]$. For example, applications for video transmission requires high system throughput (e.g capacity, area coverage) whereas energy consumption is a primary concern in process automation application. On the other hand, application of intelligent transport system needs low latency in data transmission whereas factory automation requires highly reliable communication [10]. Therefore, IoT traffic can be categorized into three domains based on their specific QoS requirements (i) energy sensitive, (ii) throughput sensitive, and (iii) highly reliable traffic. The energy sensitive traffic is defined as the traffic that can tolerate low data rate and reliability but very sensitive to power consumption. An use case of energy sensitive traffic is mMTC application. Throughput sensitive traffic is the 
one that requires high data rate and moderate reliability which basically represents eMBB service. Finally, the highly reliable traffic represents the traffic that requires high reliability in mission critical application.

While there have been significant development in digital wireless transmission technologies over the last few years, the one aimed at Massive MIMO technology holds the potential to support transmission techniques that are suitable for various IoT traffic. As described in chapter 2, massive MIMO BS uses an array of hundred or more antennas that allows coherent multiuser MIMO transmission with tens of nodes being spatially multiplexed in both UL and DL. Therefore, the area throughput is improved by the multiplexing gain but it comes at the cost of circuit power (CP). For example, multiple RF chains at BS and digital signal processing (i.e., SDMA combining/precoding) increase the $\mathrm{CP}$ power that directly affects the overall EE of the network. EE of wireless network is defined by the number of bits that can be reliably transmitted per unit of energy and it is measured in (bit/Joule) given by

$$
\mathrm{EE}=\frac{\text { Throughput }[\mathrm{bit} / \mathrm{s}]}{\text { Power consumption }[\mathrm{W}]},
$$

Therefore, to design transmission scheme for heterogeneous IoT traffic, the throughput and power consumption of massive MIMO must be properly balanced.

In this chapter, we have formulated an energy efficient traffic-oriented optimization problem for single cell Massive MIMO system that serves three different aforementioned IoT traffics. When a number of nodes belong to a specific traffic, the BS adapts its system dimensions to ensure the QoS of the corresponding traffic while maximizing the EE and based on that transmission strategies are adapted for that particular traffic. More precisely, given any set of IoT node connection requests, the proposed model simultaneously determines the optimal number of antenna and power 
allocation for serving those nodes based on their traffic characteristics and determines the modulation mode accordingly.

The rest of the chapter is outlined as follows: Section 4.2 describes the system model and assumptions. Section 4.3 illustrates the formulation and operation of proposed traffic oriented transmission scheme. Section 4.4 illustrate the simulation results in terms of number of optimum antennas, average power consumption, SE, area throughput and EE. Finally, a brief summary is given in Section 4.5.

\subsection{System Model and Assumptions}

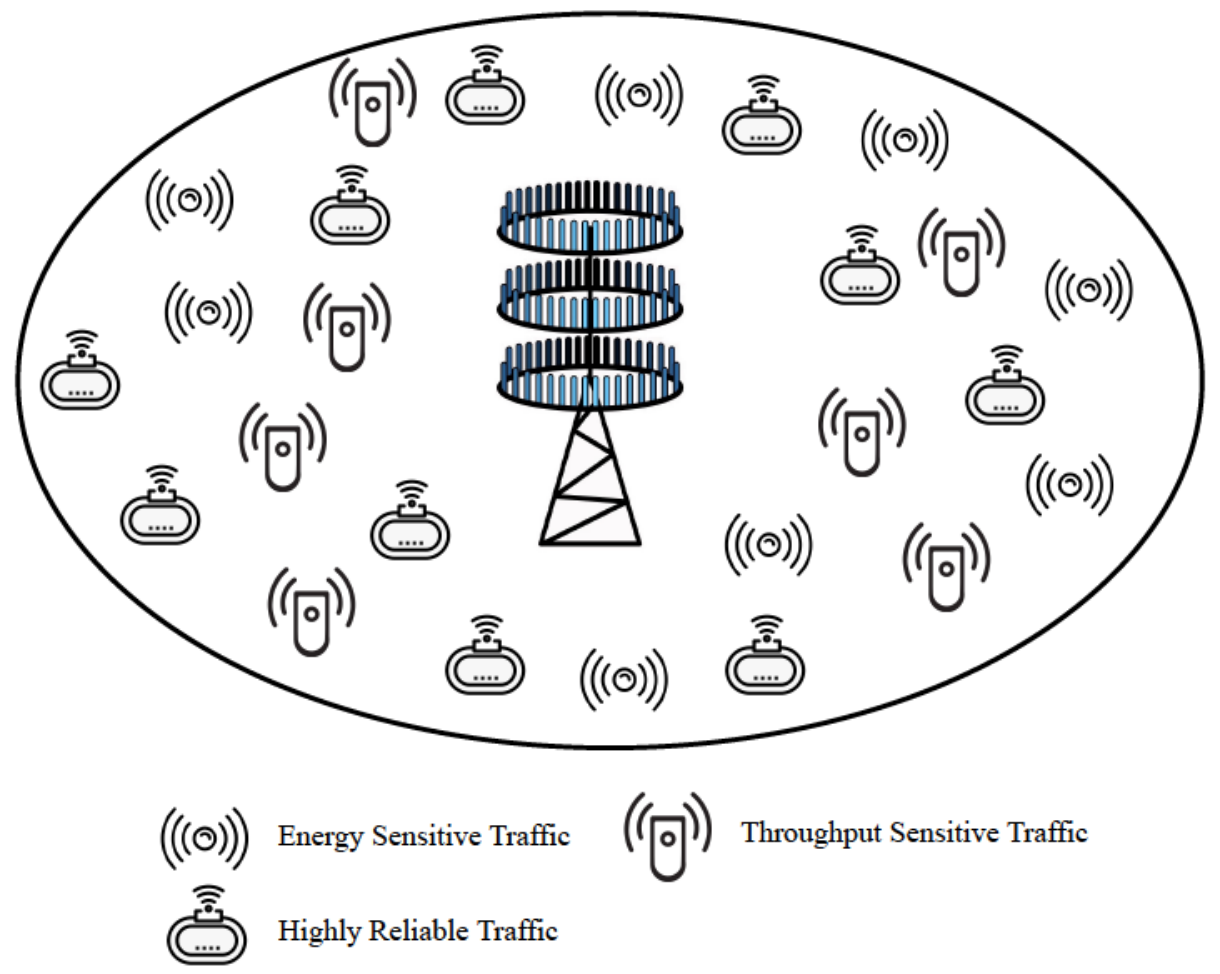

Figure 4.1: Single-cell IoT network with large number of nodes generating energy sensitive, throughput sensitive and highly reliable traffic.

We consider massive MIMO based IoT link where the BS uses an array of $M$ antennas to communicate simultaneously with a set of $K$ single antenna nodes where $M>K$. 
The nodes are assumed to be uniformly distributed in the coverage area. All the nodes in this scenario are running diverse application which is either energy sensitive application, throughput sensitive application, or highly reliable IoT application as shown in Fig. 4.1. The BS and nodes are assumed to be perfectly synchronized and operate according to the TDD mode with UL transmission ratio, $\epsilon^{u}=0.6$ and DL transmission ratio, $\epsilon^{d}=0.4$. Let us consider $U$ is the total number of complex symbols in single time-frequency coherence block which is the product of coherence frequency $B_{c}$ and coherence time $T_{c}$. The relative pilot length $\tau^{u}$ and $\tau^{d}$ indicates that UL pilot signal and DL pilot signal occupy $\tau^{u} K$ and $\tau^{d} K$ symbols, respectively. All nodes in this scenario are grouped/clustered based on their traffic type and each cluster of traffic is scheduled in round-robin fashion. The UL pilots from the scheduled group of nodes enable the BS to estimate their channels. At any transmission, the active set of nodes send its traffic information to the BS through the control channel. The BS then adapts number of antennas and select the transmission scheme for that group of nodes according to the proposed traffic-oriented scheme.

\subsubsection{Channel Model and Linear Processing}

The channel between the BS and $k_{t h}$ active node includes both large scale path loss and small scale block fading i.e the channel is constant for one coherence block and then updated independently from the circular-symmetric complex Gaussian distribution. The channel matrix $\mathbf{H}=\left[\mathbf{h}_{1}, \mathbf{h}_{2}, \ldots \mathbf{h}_{k}\right] \in \mathbb{C}^{M \times K}$ denotes instantaneous propagation channel of all nodes. The BS is assumed to have knowledge about large scale fading and estimates the small scale fading in each coherence interval. We denote the $\mathrm{UL}$ linear receive combining matrix by $\mathbf{G}=\left[\mathbf{g}_{1}, \mathbf{g}_{2}, \ldots \mathbf{g}_{k}\right] \in \mathbb{C}^{M \times K}$ and DL precoding matrix by $\mathbf{A}=\left[\mathbf{a}_{1}, \mathbf{a}_{2}, \ldots \mathbf{a}_{k}\right] \in \mathbb{C}^{M \times K}$ and set $\mathbf{G}=\mathbf{A}$. We consider Zero Forcing (ZF) as an interference suppressing linear processing scheme which gives $\mathbf{G}=\mathbf{H}\left(\mathbf{H}^{H} \mathbf{H}\right)^{-1}$. 


\subsubsection{Achievable Rate}

The average achievable rate, $R_{T}$ (bit/sec) for both UL and DL with ZF linear processing under the condition $M \geq K+1$ is given by [42]

$$
R_{T}=\left(1-\frac{\left(\tau^{u}+\tau^{d}\right) K}{U}\right) B \log (1+\gamma)
$$

Here $B$ is the transmission bandwidth of the channel, the term $1-\frac{\left(\tau^{u}+\tau^{d}\right) K}{U}$ accounts for the pilot overhead and $\gamma$ is the average SNR. To ensure reliability, we need a minimum SNR to guarantee specific BER and modulation mode. Here we have adopted Adaptive Quadrature Amplitude Modulation (M-QAM) for determining transmission rate under certain reliability. In this case, the required SNR for a target BER $\beta$ and constellation size $2^{n}$ is approximated as [52]

$$
\gamma_{T_{n}}=-\frac{2}{3} \ln (5 \beta)\left(2^{n}-1\right) ; \quad n=0,1, \ldots N,
$$

where $n$ is the modulation mode. As we can see from (4.3), for a specific modulation mode, the traffic that demands lower BER to ensure high reliability will require higher SNR.

\subsubsection{Transmit Power}

We have adopted equal power control method to guarantee equal bit rate to the $K$ nodes that belongs to the same traffic. Let $\mathbf{P}^{u}=\operatorname{diag}\left(p_{1}^{u}, p_{2}^{u}, \ldots, p_{k}^{u}\right)$ denote the UL transmitted power by node $i$ for $i=1,2, \ldots K$. It follows that the UL power allocation vector $\mathbf{p}^{u}=\left[p_{1}^{u}, p_{2}^{u}, \ldots, p_{k}^{u}\right]^{T}$ and DL power allocation vector $\mathbf{p}^{d}=\left[p_{1}^{d}, p_{2}^{d}, \ldots, p_{k}^{d}\right]^{T}$ must satisfy the condition [53] 


$$
\begin{aligned}
& \mathbf{p}^{u}=\sigma^{2}\left(\mathbf{D}^{u}\right)^{-1} 1_{K}, \\
& \mathbf{p}^{d}=\sigma^{2}\left(\mathbf{D}^{d}\right)^{-1} 1_{K},
\end{aligned}
$$

where $\sigma^{2}$ denotes the noise variance at the $k_{t h}$ node in (Joule/symbol) and the $(1, \mathrm{k})$ th element of both $\left(\mathbf{D}^{u}, \mathbf{D}^{d}\right) \in \mathbb{C}^{K \times K}$ is given by [42]

$$
\begin{aligned}
\mathbf{D}^{u} & =\frac{\left|\mathbf{g}_{k}^{H} \mathbf{h}_{k}\right|^{2}}{\left.2^{(R / B-1}\right)\left\|\mathbf{g}_{k}\right\|^{2}} \\
\mathbf{D}^{d} & =\frac{\left|\mathbf{h}_{k}^{H} \mathbf{a}_{k}\right|^{2}}{\left.2^{(R / B-1}\right)\left\|\mathbf{a}_{k}\right\|^{2}}
\end{aligned}
$$

Due to the duality of TDD mode, the total UL and DL transmit power are the same if the same processing scheme is used for transmit precoding and receive combining. The only difference is the power amplifier efficiency of nodes and the BS and the fraction of transmission (i.e, $\epsilon_{u}$ and $\epsilon_{d}$ ). The transmit power consumption $P_{T}$ is given as follows where the UL and DL parameters are denoted using superscripts.

$$
\begin{aligned}
& P_{T}^{u}=\frac{B \epsilon^{u}}{\eta^{K}} \mathbb{E}\left\{\mathbf{1}_{K}^{T} \mathbf{p}_{k}^{u}\right\} \\
& P_{T}^{d}=\frac{B \epsilon^{d}}{\eta^{B S}} \sum_{k=1}^{K} \mathbb{E}\left\{p_{k}^{d}\right\} .
\end{aligned}
$$

\subsubsection{Circuit Power}

We define $P_{c p}$ as the total circuit power spent by the BS and IoT nodes. Circuit power consumption of the BS consists of a fixed part (e.g., control signal, backhaul, DC-DC conversion) and a dynamic part which depends on the number of active antennas $M$, number of nodes $K$, and the channel gain. We have adopted the circuit power model 
as [42] where the total circuit power consumed in total UL and DL transmission is given by

$$
P_{c p}=\alpha_{1} R_{T}+\alpha_{2}+K P_{t c}^{K}+M P_{t c}^{B S}+P_{C E}+P_{l p}
$$

where $\alpha_{1}$ accounts for coding, decoding, and backhaul power consumption and $R_{T}$ is calculated using (4.2). The second term $\alpha_{2}$ is the constant power consumption for site cooling, control signaling, and frequency synthesis at the BS. The term $P_{t c}$ represents the transceiver power consumption by the nodes and BS denoted by the superscript. $P_{C E}$ stands for the BS channel estimation's power consumption and is given by

$$
P_{C E}=\frac{B K^{2}}{U}\left(\frac{2 M \tau^{u}}{\Psi_{B S}}+\frac{4 \tau^{d}}{\Psi_{K}}\right)
$$

where $\Psi_{B S}$ and $\Psi_{K}$ denote the computational efficiency of the BS and the $K$ nodes, respectively. Finally, $P_{l p}$ accounts for the power consumed for linear processing. For $\mathrm{ZF}$ precoding, $P_{l p}$ is given by

$$
\begin{aligned}
P_{l p} & =\frac{2 B M K}{\Psi_{B S}}\left(1-\frac{\left(\tau^{u}+\tau^{d}\right) K}{U}\right) \\
& +\frac{B}{U}\left(\frac{K^{3}}{3 \Psi_{B S}}+\frac{3 M K^{2}+M K}{\Psi_{B S}}\right)
\end{aligned}
$$

It is important to note that the circuit power consumption is a function of number of antennas $(M)$ and number of nodes $(K)$ which means more antennas and nodes will consume more power. Therefore, while designing an energy efficient transmission scheme for the IoT traffic we need to optimize the number of antennas depending on the number of active nodes in the network to better utilize the resources. The description of each parameter of circuit power consumption is shown in Table 4.1. 
Table 4.1: Reference Parameter

\begin{tabular}{|c|c|}
\hline Parameter & Value \\
\hline Coding, decoding, and backhaul, $\alpha_{1}$ & $1.15 \times 10^{-9} \mathrm{~W} /(\mathrm{Gbit} / \mathrm{s})$ \\
Static power consumption, $\alpha_{2}$ & $20 \mathrm{~W}$ \\
Transceiver chain at BS, $P_{t c}^{B S}$ & $1 \mathrm{~W}$ \\
Transceiver chain of nodes, $P_{t c}^{K}$ & $0.1 \mathrm{~W}$ \\
DL transmission, $\epsilon^{d}$ & 0.4 \\
UL Transmission, $\epsilon^{u}$ & 0.6 \\
Pilot length at UL and DL $: \tau^{u}, \tau^{d}$ & 1 \\
Computational Efficiency at BS, $\Psi_{B S}$ & $12.8 \mathrm{Gflops} / \mathrm{W}$ \\
Computational Efficiency at nodes, $\Psi_{K}$ & $5 \mathrm{Gflops} / \mathrm{W}$ \\
Power amplifier efficiency at BS, $\eta^{B S}$ & 0.39 \\
Power amplifier efficiency at nodes, $\eta^{K}$ & 0.3 \\
\hline
\end{tabular}

\subsection{Traffic-Oriented Transmission Scheme}

In this section, we aim at finding the optimal SNR and the corresponding number of antennas to select the transmission scheme for each of the heterogeneous IoT traffics. The optimal SNR under different traffic constraint allows to select the modulation scheme ensuring low power consumption for energy sensitive traffic, high bit rate for throughput sensitive traffic, and better error performance for the highly reliable traffic. Let us denote the BER for energy, throughput, and highly reliable traffics by $\beta_{\mathrm{E}}$, $\beta_{\mathrm{T}}$, and $\beta_{\mathrm{R}}$, respectively. For the energy sensitive traffic, we enable a communication with the lowest modulation mode. On the other side, for throughput sensitive traffic the communication takes place in high SNR region. Finally, for the highly reliable traffic, the operating region is set to satisfy BER of $\beta_{R}$. 


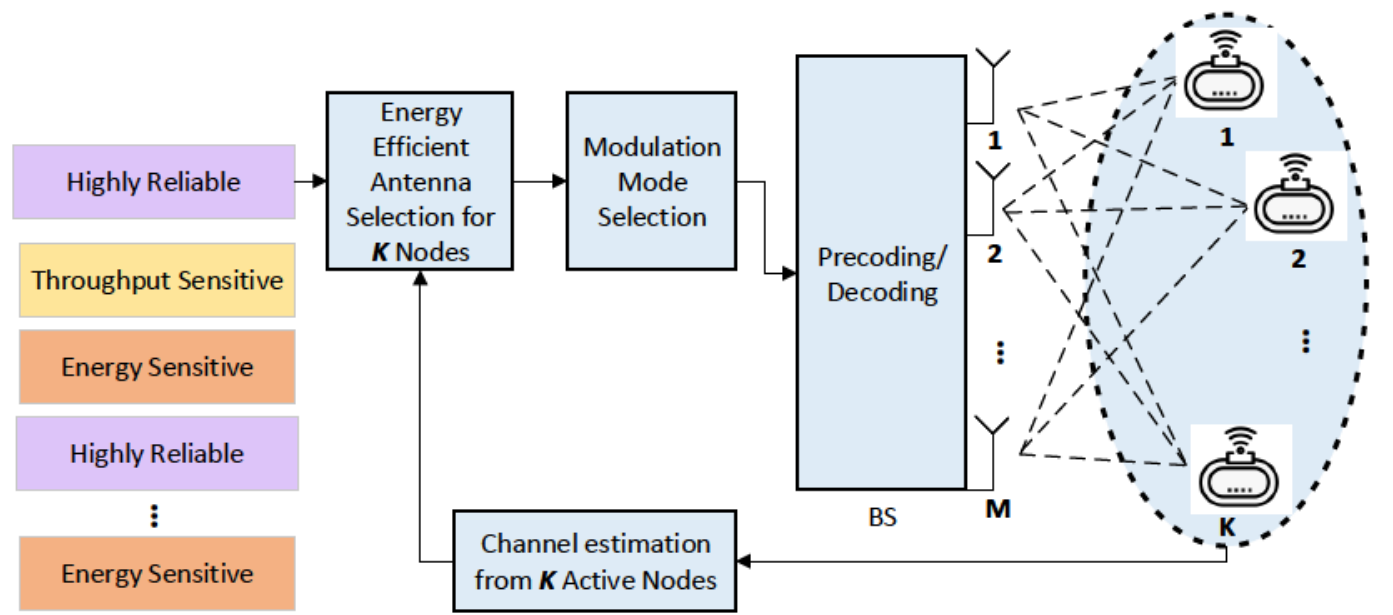

Figure 4.2: Operational diagram of traffic adaptive transmission scheme.

The optimal system parameter should maintain the EE of the overall wireless system for every IoT traffic it encounters. Adding more $M$ and $K$ antennas will result in higher coverage (i.e, sum SE) but at the same time, it will cost power consumption from the connected transceiver chains. Therefore, an energy efficient design allows us to better utilize the resources and optimize the operational cost. As described in the previous sections, circuit power consumption of massive MIMO system is a function of $M$ and $K$ which means increasing the number of antennas and number of users will result in high power consumption. Therefore, an energy efficient transmission scheme should be adopted in every transmission that can adapt the number of antennas with the number of active nodes based on their traffic type. For this reason, we are interested to find the optimal operating point for any number of active nodes of the three different IoT traffic in the network that will maximize the EE under the corresponding traffic's QoS constraints. The optimization problem to maximize the overall EE in a multi-user setting for both UL and DL is formulated as follows 


$$
\left(M^{*}, \gamma^{*}\right)=\operatorname{argmax} \mathrm{EE}=\frac{\sum_{k=1}^{K} R_{T}(\gamma)}{\left(P_{T}^{u}+P_{T}^{d}\right)+P_{c p}(M, K, \gamma)},
$$

Subject to

$$
\begin{aligned}
& \gamma \leq \gamma_{T_{E}} \quad \text { for energy sensitive traffic, } \\
& \gamma \geq \gamma_{T_{T}} \quad \text { for throughput sensitive traffic, } \\
& \gamma \geq \gamma_{T_{R}} \quad \text { for highly reliable traffic, }
\end{aligned}
$$

where $\gamma_{T_{E}}, \gamma_{T_{T}}$ and $\gamma_{T_{R}}$ are given by

$$
\begin{array}{cc}
\gamma_{T_{E}}=\gamma_{T_{2}} & \text { for } \beta_{E}, \\
\gamma_{T_{T}}=\gamma_{T_{4}} & \text { for } \beta_{T}, \\
\gamma_{T_{R}}=\gamma_{T_{1}} & \text { for } \beta_{R},
\end{array}
$$

where $\gamma_{T_{2}}, \gamma_{T_{4}}$ and $\gamma_{T_{1}}$ are calculated using (4.3). The numerator in eq. (4.13) accounts for the achievable rate by multiplexing $K$ nodes and the denominator stands for the total power consumption required to achieve same level of performance to those nodes. This power consumption comes from both transmit power and circuit power defined in eq. (4.4) and (4.10). Note that (4.14a) restricts the rate transmission to lowest modulation mode so that the low power node use less energy. Eq. (4.14b) ensures that when the BS will serve the throughput sensitive nodes, it will serve with high modulation mode since the optimal SNR is set to achieve high SE. Finally, eq. (4.14c) sets the modulation threshold for a lower BER to ensure high reliability for highly reliable traffic and the constraints is applied for low constellation size.

Eq. (4.13) is a constrained optimization problem where we want to find optimal number of antenna $M$ for a given number of nodes $K$. Since we are considering 
ZF linear processing, we need to set $M \geq K+1$. At first, for any number of scheduled nodes $(K)$ bearing identical traffic, we apply equal power allocation method to guarantee an equal SNR which is feasible for all nodes. After that, we find the optimal feasible SNR $\left(\gamma^{*}\right)$ by gradient decent method that satisfies the corresponding traffic's SNR constraint in (4.14) and maximizes EE in the objective function (4.13). This optimization is executed for every node $(K)$ with all possible combination of antennas $(M)$. We therefore, use brute force method to search over all the values of $M$ that gives maximum EE value for that particular number of nodes $(K)$. The number of antennas $(M)$ that globally maximizes EE, is the optimum $M^{*}$. After we find the optimum number of antennas and corresponding power allocation, we determine the modulation scheme that needs to be applied for the individual IoT traffic. This way in every transmission, the proposed traffic adaptive transmission scheme adapts the number of antennas and modulation mode based on the traffic type. The detailed algorithm is shown in Algorithm 1 and the operation is shown in Fig. 4.2. 


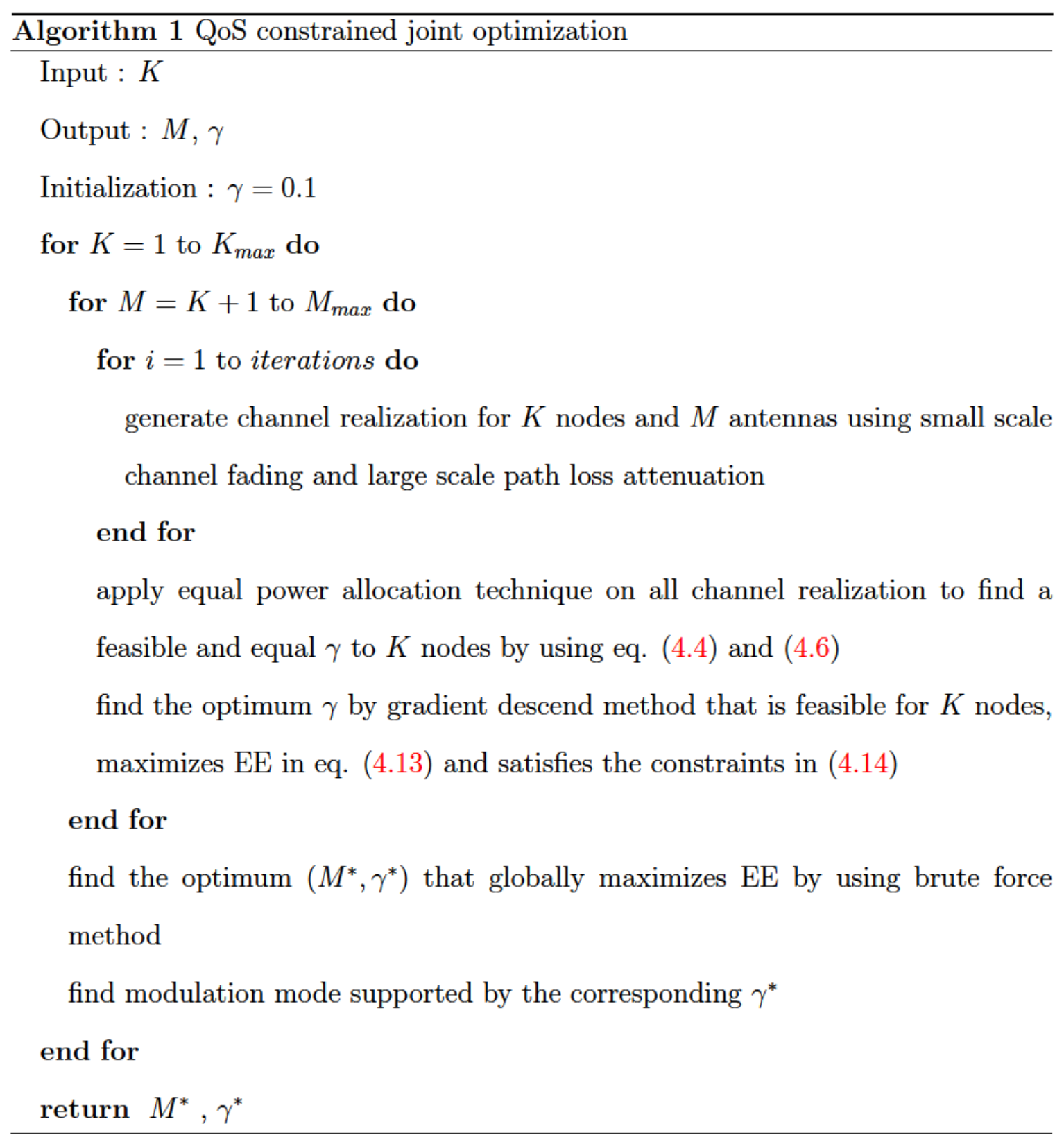

\subsection{Numerical results}

This section provides numerical results using Monte Carlo simulations with random user locations considering both large scale and small scale fading. We consider a single-cell scenario where the BS is equipped with 126 antennas and can serve three 
group of traffics, each of them contains up to 50 nodes. The simulation is performed with respect to number of nodes that acts as a design parameter for individual IoT traffic. The simulation parameters are given in Table 4.2 and was performed using MATLAB.

Table 4.2: Simulation Parameter

\begin{tabular}{|c|c|}
\hline Simulation Parameter & Value \\
\hline Maximum number of antenna, $M_{\max }$ & 126 \\
Maximum number of nodes, $K_{\max }$ & 50 \\
Number of iterations & 100 \\
Cell radius, $d_{\max }$ & $250 \mathrm{~m}$ \\
Minimum distance, $d_{\min }$ & $35 \mathrm{~m}$ \\
Path loss at distance $d$ & $\frac{10^{-3.53}}{\|d\|^{3.76}}$ \\
Transmission bandwidth, $B$ & $20 \mathrm{MHz}$ \\
Channel coherence time, $T_{c}$ & $10 \mathrm{~ms}$ \\
Channel coherence bandwidth, $B_{c}$ & $180 \mathrm{KHz}$ \\
Channel coherence interval, $U$ & $1800 \mathrm{symbols}$ \\
Noise power & $-96 \mathrm{dBm}$ \\
BER of energy and throughput sensitive traffic, $\beta_{E}=\beta_{T}$ & $10^{-3}$ \\
BER of highly reliable traffic, $\beta_{R}$ & $10^{-6}$ \\
\hline
\end{tabular}

\subsubsection{Optimal Number of Antenna}

Fig. 4.3 illustrates the optimal antenna-node ratio $(M / K)$ i.e, the number of antennas that should be proportionally increase with the number of nodes to maximize EE for all types of traffic under consideration. For every traffic, it is achieved by finding their optimal SNR region under the constraints in eq. (4.14) and then finding the number of antennas $(M)$ that will maximize the objective function in (4.13). Therefore the difference in number of antennas is due to different operating region constrains (i.e, 
the SNR constraints) applied to the individual traffic. Since the SNR constraints is set to be higher for the throughput sensitive and highly reliable traffics for higher throughput and reliability respectively, they require more antennas than the energy sensitive traffic. Fig. 4.3 depicts that the number of antennas to serve 40 energy sensitive nodes is 58 whereas 76 and 81 antennas need to be active for serving the same number of highly reliable and throughput sensitive nodes, respectively.

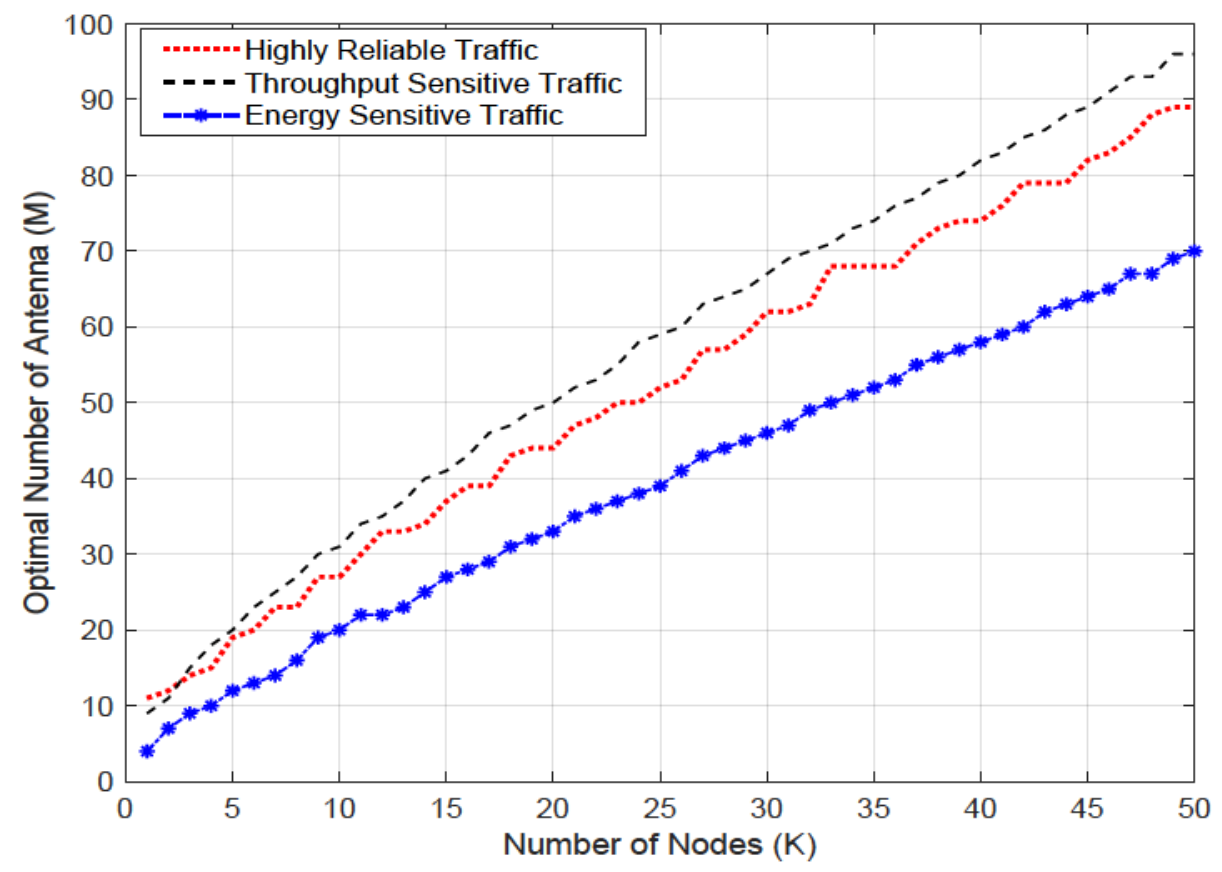

Figure 4.3: Optimal number of antennas at EE maximizing point for different traffics.

\subsubsection{Average Transmit Power}

Fig. 4.4 illustrates the average UL transmit power per IoT node for different $K$ (using the corresponding optimal $M$ ) that maximizes EE under QoS constraint . We see that the throughput sensitive traffic and highly reliable traffic need to operate with high power consumption. This is because throughput sensitive traffic need to spend more power for high data rate whereas highly reliable traffic requires that to 
ensure high reliability. We also observe that for all types of traffic, the transmit power consumption per node decreases with the number of nodes $K$. This is the result of array gain which means the proportional increase in number of antennas with the number of nodes allows the array to collect more energy from the desired signal. As a result, the higher array gain (results from additional antennas in Fig. 4.3) improves SNR and allows the nodes to use less power in transmission. Fig. 4.4 also shows the variation of power level for different traffics. For example, when 40 throughput sensitive and highly reliable nodes need to transmit data, they will be consuming $236 \mathrm{~mW}$ and $197 \mathrm{~mW}$ power, respectively. In contrast, the same number of energy sensitive nodes will use $72 \mathrm{~mW}$. This is again because of the different SNR regimes of different traffics.

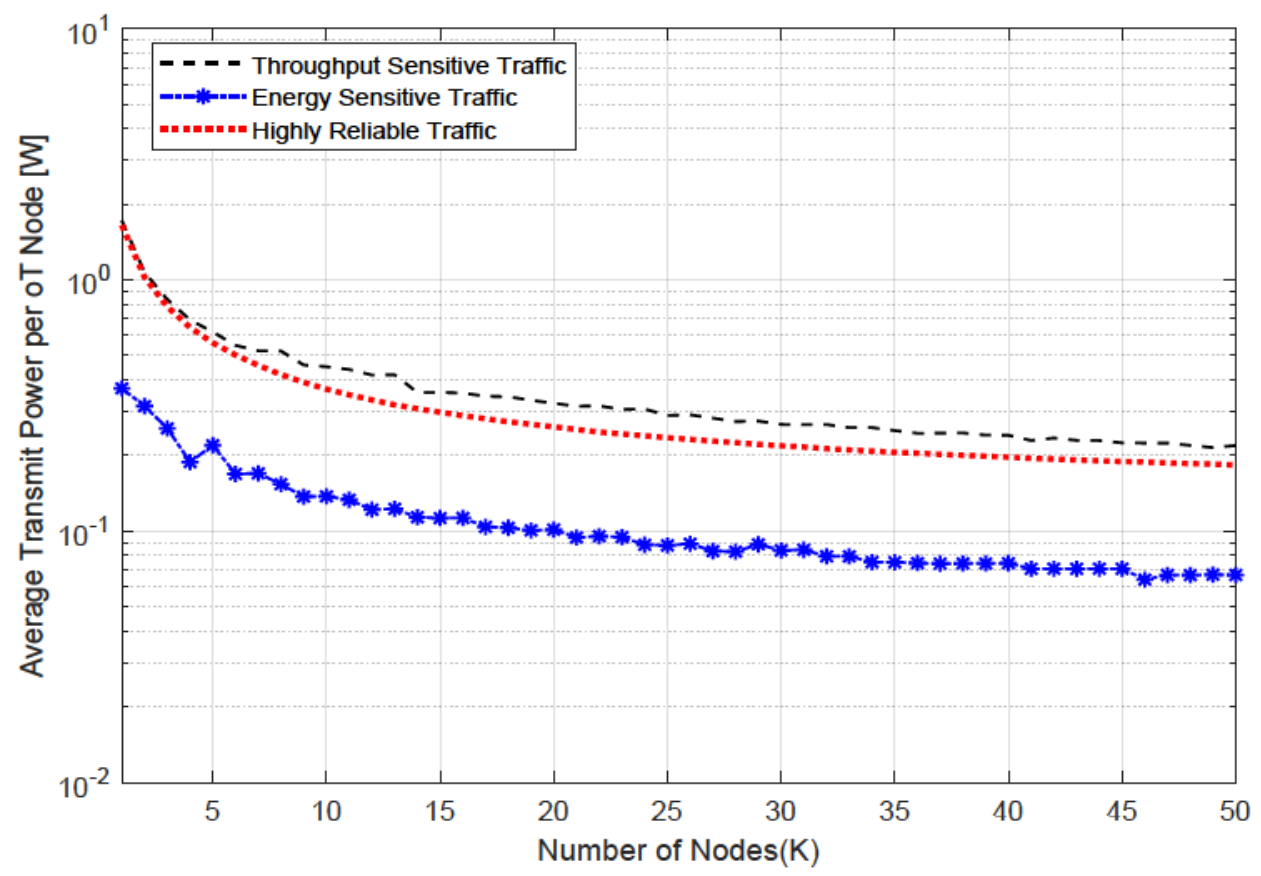

Figure 4.4: Average transmit power per node at EE-maximization solution. 


\subsubsection{Spectral Efficiency}

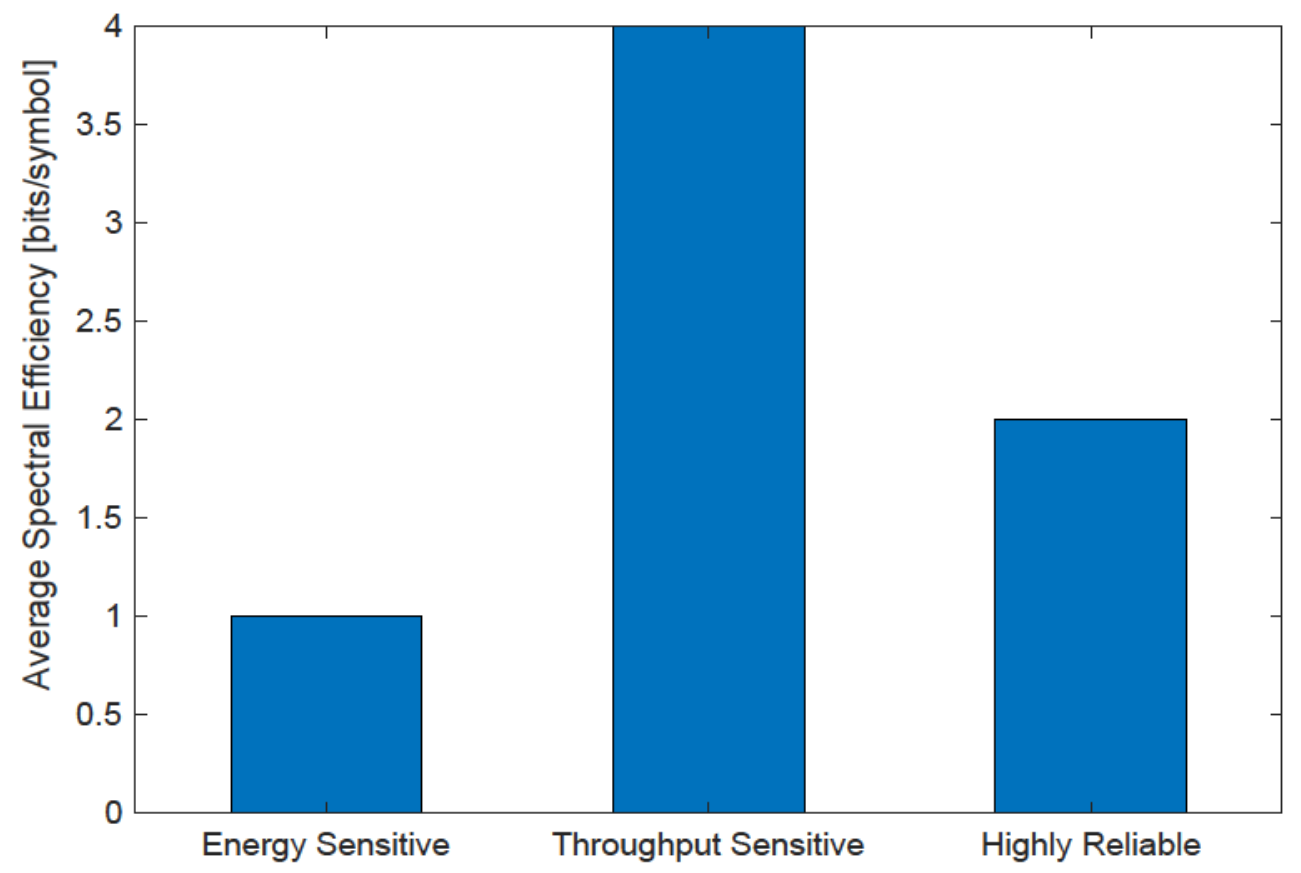

Figure 4.5: Achievable spectral efficiency for different IoT traffic.

Fig. 4.5 compares the achievable SE in bits per symbol that allows us to select the modulation scheme for different IoT traffics. This result is achieved after finding the optimal number of antennas and optimal SNR. As mentioned in 4.3 the scheme applies equal power control technique to guarantee a given feasible SNR to all nodes and then finds the optimal feasible SNR regime that maximizes EE in eq. (4.13) and satisfies QoS constraints in eq. (4.14). This way, the SNR is calculated for every possible combination of $K$ and $M$ but out of all the combinations, the optimum SNR that is found from the optimal antenna-node ratio is used for determining the modulation mode. Fig. 4.5 shows that our proposed energy efficient transmission scheme allows lowest SE of only 1 bit per symbol for the energy sensitive traffic because of the rate constraint applied in the optimization problem. This also means that this traffic will be allowed to communicate with binary phase shift keying (BPSK) using the BER of 
$10^{-3}$ to minimize power consumption as shown in Fig. 4.4. The optimal modulation mode for highly reliable traffic is found to be 2 bits per symbol which means the energy efficient modulation mode for this type of traffic is QPSK under low BER of $10^{-6}$. As we have seen in Fig. 4.3 and Fig. 4.4, this higher reliability comes at the cost of high antenna selection and transmit power respectively. Finally, the energy efficient modulation mode for throughput sensitive traffic is found to be 16-QAM or 4 bits per symbol under the BER of $10^{-3}$ which is also an outcome of high number of antennas and high power consumption.

\subsubsection{Area Throughput}

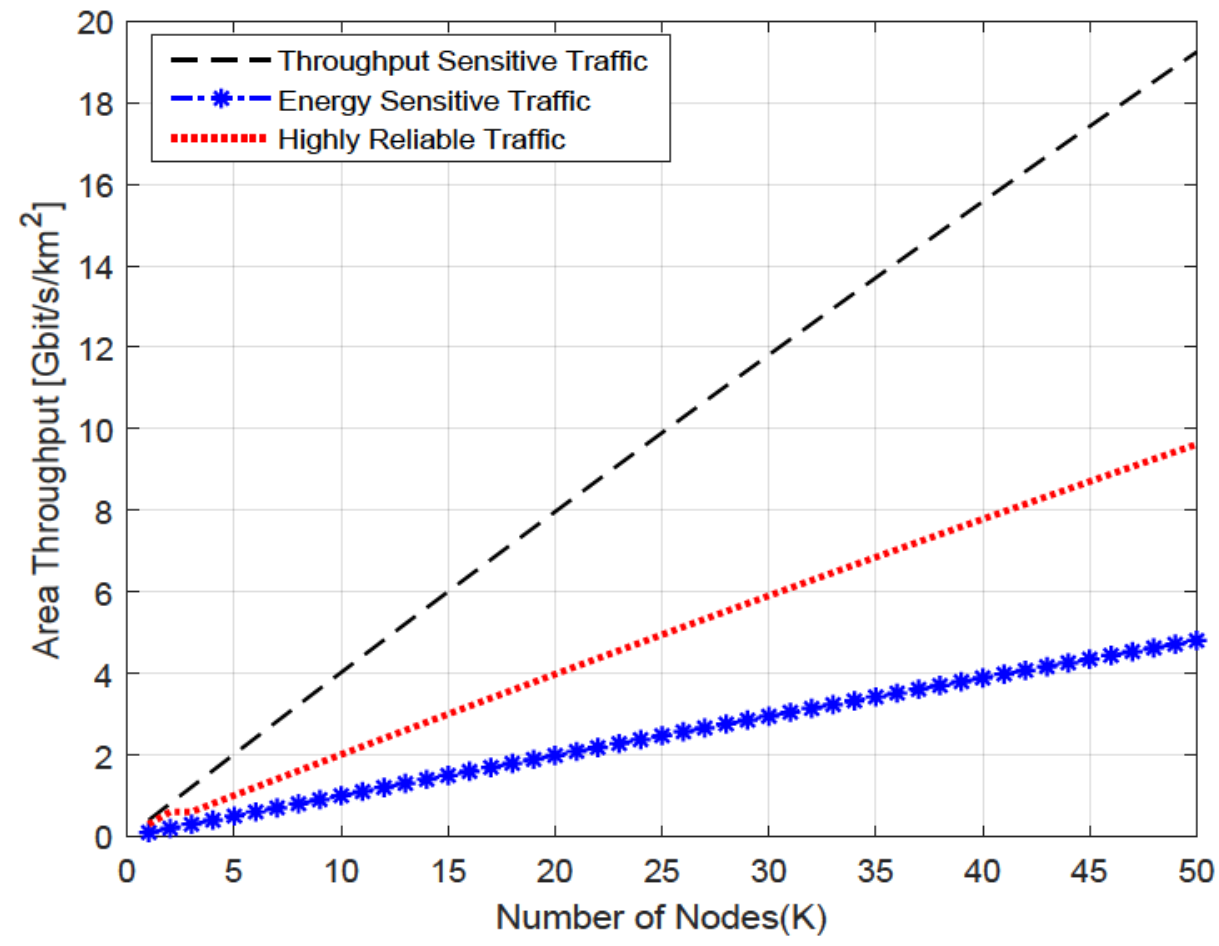

Figure 4.6: Area throughput at EE maximizing solution.

Fig. 4.6 shows the area throughput in $\left(G b i t / s / \mathrm{km}^{2}\right)$ that maximizes the EE for different number of nodes $K$ that will be served simultaneously. The EE maximizing 
throughput is achieved using transmission bandwidth of $20 \mathrm{MHz}$ which is typically used in current LTE network. For all the considered traffic, higher number of nodes results in higher the aggregated data rate. This is the impact of spatial multiplexing gain that results in proportional increase in sum SE with the number of nodes. As expected, the throughput sensitive traffic has the highest throughput because of the high SE as shown in Fig. 4.5. The other two traffic is also following the same trend of SE. Fig. 4.6 also indicates that using only 40 antennas from BS as shown in Fig. 4.3, 25 energy sensitive nodes can be served simultaneously with total data rate of $2.5 \mathrm{Gbit} / \mathrm{s}$. On the other hand, the energy efficient solution in Fig. 4.3 yields that 52 and 60 antennas are sufficient for providing total data rate of $5 \mathrm{Gbit} / \mathrm{s}$ and 10 Gbit/s per kilometer of area, to the same (i.e, 25) number of highly reliable nodes and throughput sensitive nodes, respectively. 


\subsubsection{Energy Efficiency}

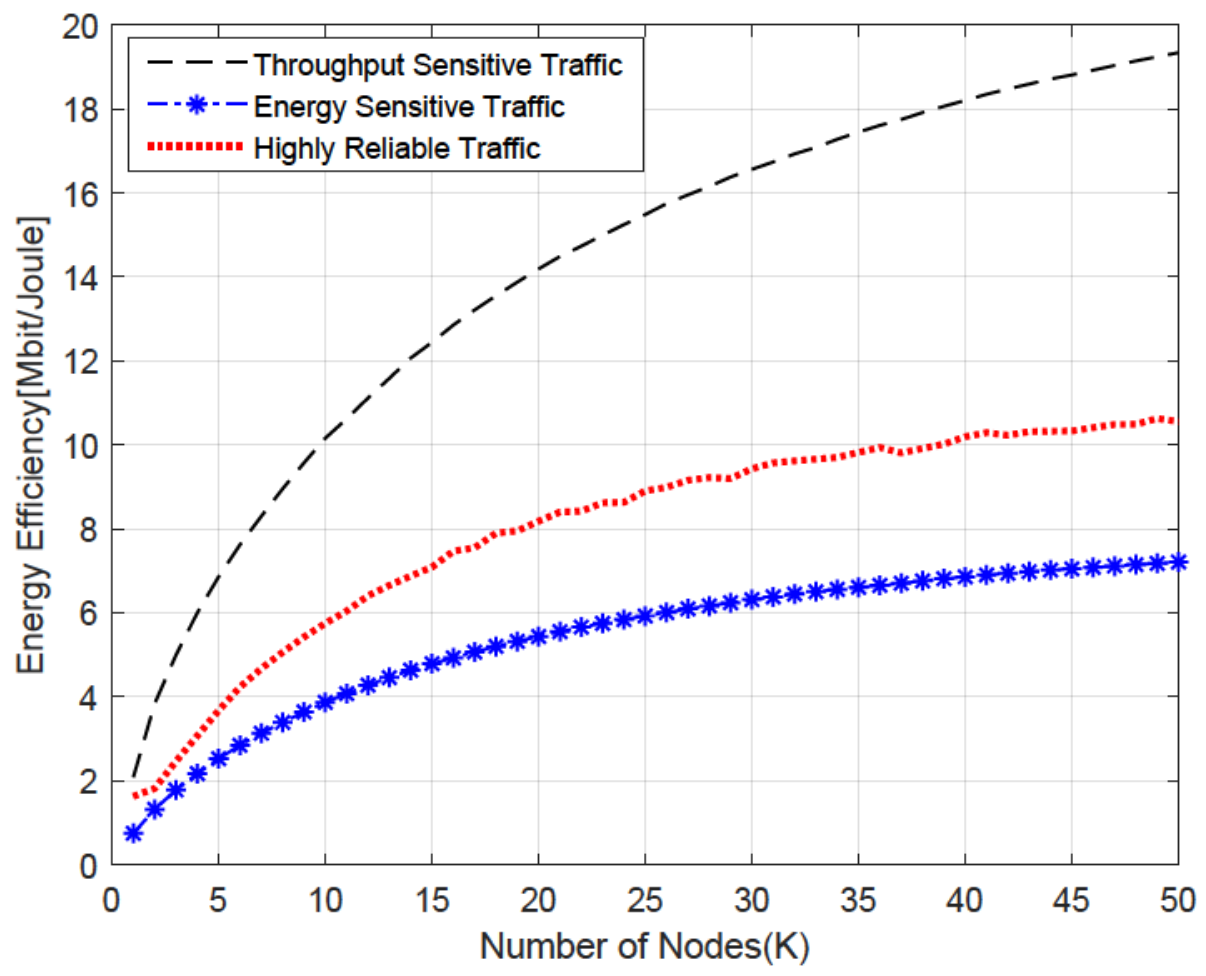

Figure 4.7: Maximum energy efficiency using optimal number of antennas.

Fig. 4.7 shows the EE $(M b i t / J)$ for different traffic under the QoS constraints. The EE for all types of traffic increases significantly with the number of nodes. This is attributed to the multiplexing and array gain of massive MIMO that increases with the number of nodes and results in high throughput as shown in Fig. 4.6 and low transmit power in Fig. 4.4, respectively. As expected, the throughput sensitive traffic shows highest EE because the proposed scheme allows this traffic to operate in high SNR region such that it can maintain higher throughput. As the throughput increases with number of nodes, the EE also increases. The trend of highly reliable traffic depicts that although energy efficient solution enables this traffic to operate in high SNR, that SNR is utilized for ensuring low BER. Therefore, the rate is compromised and it shows lower EE compared to throughput sensitive traffic. Finally, the energy 
sensitive traffic shows the least EE as it transmits with low bit rate which impacts the throughput. Although it achieves low power consumption, but the ratio between the throughput and power consumption is lower than other two traffics.

\subsection{Conclusion}

In this study, we aim at designing a traffic-oriented energy efficient Massive MIMO system that can optimally scale the number of antennas with the number of active nodes in the network and adapt the transmission scheme with the traffic type. The results show that by adapting the number of antennas with proper modulation mode, the heterogeneous IoT traffic can be accommodated in a common wireless transmission infrastructure. Our study also shows that the energy sensitive traffic should be communicated with lowest modulation mode of BPSK under a moderate BER of $10^{-3}$ since this type of application represents low power low rate sensor communication that can tolerate low SE. On the otter hand, the throughput sensitive nodes should be served with higher number of antennas and higher order modulation mode to guarantee high SE of 4 bits per symbol. Finally, The highly reliable traffic needs to be served with larger number of antennas to support high reliability with BER of $10^{-6}$ and the transmission scheme should be the modulation mode of QPSK. By taking advantage of massive MIMO technology, the performance of the proposed scheme is shown to be energy efficient to all IoT traffics. 


\section{Chapter 5}

\section{Conclusions and Future Work}

\subsection{Conclusions}

IoT introduces a paradigm shift to wireless communications. To effectively support various applications, future wireless IoT systems need to optimize their transmission strategies for a large number of devices with diverse service requirements while providing wide coverage and maintaining energy efficiency. Traditional transmission technologies are typically designed with the goal of enhancing or approaching the capacity limits of wireless channels which works well for many IoT applications that require high volume data transfer but are not suitable for other types of IoT applications that have different performance requirements. Therefore, the conventional transmission technique will be power expensive for many low-power IoT devices if such heterogeneity is not taken into account. In this thesis, we presented two sets of adaptive transmission techniques that consider the device and traffic heterogeneity respectively.

First, an adaptive transmission technique named HS scheme is proposed for a simple IoT network operating over SISO model. This scheme takes the energy level of the IoT device into consideration when deciding on the transmit power and the 
modulation mode at every transmission. The scheme shows how the combination of low power and high power devices affects the power usage ratio and spectral efficiency compared to the traditional systems that target capacity enhancement. Finally, the HS scheme has many possible IoT applications where battery level consideration is very crucial. For example, renewable energy-based IoT applications where the devices either run by the battery power and directly powered by a renewable source. In such a scenario, the HS scheme is capable to adapt its transmission scheme according to the nodes' battery level.

Multi-antenna technology offers better performance over a single antenna system in terms of coverage, link reliability, and capacity. Therefore, in chapter 4 we focus on a massive MIMO driven IoT network. In massive MIMO, while substantial SE gain can be achieved by multiplexing $K$ nodes, similar results can not be achieved for $\mathrm{EE}$ as adding more antennas will result in more power consumption from the circuit components at BS. Also, the number of connected nodes in the network varies as the transmissions usually occur sporadically. Therefore, in every transmission an optimal antenna-node ratio $(M / K)$ is required that will allow the BS to adapt the number of antennas with the number of active nodes and operate with maximum EE. As IoT system deals with different traffics, we aim to find the optimal antenna-node ratio $(M / K)$ with proper transmission scheme for three different IoT traffic, named as energy sensitive traffic, throughput sensitive traffic and highly reliable traffic. For this purpose, we have formulated an energy efficient optimization problem considering the traffic constraints. Simulation results show that the proposed model can proportionally adapt the number of antennas with the number of IoT nodes in an energy efficient way. Also, it finds out energy efficient modulation mode that needs to be applied for the considered IoT traffic. 


\subsection{Future Work}

The insights attained in this open demanding future research direction, including, but not limited to are:

- In this thesis, our traffic adaptive scheme in Massive MIMO system considers that in each transmission, the BS is serving the group of IoT nodes that are generating the same type of traffic. In future work, we are planning to investigate the case, when the BS simultaneously serves the nodes from different traffics.

- Taking the delay into account is another approach to be considered. In the traffic adaptive scheme, we have considered the round-robin scheduling as a fair scheduling technique. In future work, we can prioritize the access of the nodes that are running mission-critical applications to reduce their wait time and delay.

- This thesis considers the rate expression that is valid for traditional long packet transmission. In IoT, we also need to deal with short packet transmission where the traditional information theory for the large payload is not efficient. In the future model, we can investigate the performance of the proposed schemes for both small and large payloads.

- In this thesis, the hybrid scheme considers the energy level of the nodes and the traffic adaptive scheme considers the traffic heterogeneity of IoT applications. In future works, we are planning to develop a scheme that will simultaneously consider power heterogeneity and traffic heterogeneity of the nodes.

- We can investigate and compare our results for different processing schemes for example MMSE, MR, and ZF schemes to identify which processing scheme works best for individual traffic. 
- This thesis considers the IoT nodes as single antenna devices. However, IoT devices can have more than one antenna. Therefore, the combination of both single antenna and multi-antenna devices in a massive MIMO system is an important research topic to be considered.

- In our thesis, the performance of the adaptive transmission schemes is shown for a single-cell scenario. We can consider upgrading the system model from single cell to multi-cell scenario. In that case, we need to consider the inter-cell interference.

- Incorporating machine learning techniques to Massive MIMO is another interesting topic of investigation. Machine learning can help in adapting the antennas by predicting and analyzing the load variation of individual traffic. 


\section{List of Publications}

1. C. S. Ferdowsy, Z. Bouida, and Mohamed Ibnkahla, "Energy and Spectral Efficient Scheduling for Heterogeneous IoT Sensor Nodes," 2020 IEEE Eighth International Conference on Communications and Networking (ComNet'20), Hammamet, Tunisia, October 2020.

2. C. S. Ferdowsy, Z. Bouida, and Mohamed Ibnkahla, "Traffic Adaptive Transmission Schemes for the Internet of Things," 2020 International Conference on Communications, Signal Processing, and their Applications (ICCSPA'20), Sharjah, United Arab Emirates, November 2020. 


\section{List of References}

[1] A.-S. Bana, E. De Carvalho, B. Soret, T. Abrão, J. C. Marinello, E. G. Larsson, and P. Popovski, "Massive MIMO for Internet of Things Connectivity," Physical Communication, vol. 37, p. 100859, 2019.

[2] S. Li, L. Da Xu, and S. Zhao, "5G Internet of Things: A survey," Journal of Industrial Information Integration, vol. 10, pp. 1-9, 2018.

[3] S. Cisco, "Cisco visual networking index: Global mobile data traffic forecast update, 2017-2022," Cisco Public Information: San Jose, CA, USA, 2017.

[4] I. WP5D, "Minimum requirements related to technical performance for IMT2020 radio interface (s)," 2017.

[5] J. Ni, X. Lin, and X. S. Shen, "Efficient and Secure Service-Oriented Authentication Supporting Network Slicing for 5G-Enabled IoT," IEEE Journal on Selected Areas in Communications, vol. 36, no. 3, pp. 644-657, 2018.

[6] H. Zhang, N. Liu, X. Chu, K. Long, A. Aghvami, and V. C. M. Leung, "Network Slicing Based 5G and Future Mobile Networks: Mobility, Resource Management, and Challenges," IEEE Communications Magazine, vol. 55, no. 8, pp. 138-145, 2017.

[7] P. Popovski, K. F. Trillingsgaard, O. Simeone, and G. Durisi, "5G Wireless Network Slicing for eMBB, URLLC, and mMTC: A Communication-Theoretic View," IEEE Access, vol. 6, pp. 55765-55779, 2018.

[8] H. Ji, S. Park, J. Yeo, Y. Kim, J. Lee, and B. Shim, "Ultra-reliable and lowlatency communications in 5G downlink: Physical layer aspects," IEEE Wireless Com., vol. 25, pp. 124-130, JUNE 2018.

[9] I. T. S. Sector, "Recommendation itu-t y. 2060: Overview of the internet of things," Series Y: Global information infrastructure, internet protocol aspects 
and next-generation networks-Frameworks and functional architecture models. Retrieved from https://www. itu. int/rec/T-REC-Y, pp. 2060-201206, 2012.

[10] P. Schulz et al., "Latency critical IoT applications in 5G: Perspective on the design of radio interface and network architecture," IEEE Communications Magazine, vol. 55, pp. 70-78, February 2017.

[11] M. Series, "Imt vision-framework and overall objectives of the future development of imt for 2020 and beyond," 2015.

[12] M. Bennis, M. Debbah, and H. V. Poor, "Ultrareliable and low-latency wireless communication: Tail, risk, and scale," Proceedings of the IEEE, vol. 106, pp. 1834-1853, Oct 2018.

[13] M. Shafi, A. F. Molisch, P. J. Smith, T. Haustein, P. Zhu, P. De Silva, F. Tufvesson, A. Benjebbour, and G. Wunder, "5G: A tutorial overview of standards, trials, challenges, deployment, and practice," IEEE Journal on Selected Areas in Com., vol. 35, pp. 1201-1221, June 2017.

[14] C. Bockelmann, N. Pratas, H. Nikopour, K. Au, T. Svensson, C. Stefanovic, P. Popovski, and A. Dekorsy, "Massive machine-type communications in 5G: physical and MAC-layer solutions," IEEE Communications Magazine, vol. 54, pp. 59-65, Sep. 2016.

[15] F. Rusek, D. Persson, B. K. Lau, E. G. Larsson, T. L. Marzetta, O. Edfors, and F. Tufvesson, "Scaling up MIMO: Opportunities and challenges with very large arrays," IEEE Signal Processing Magazine, vol. 30, pp. 40-60, Jan 2013.

[16] D. Gesbert, M. Kountouris, R. W. Heath, C. Chae, and T. Salzer, "Shifting the MIMO paradigm," IEEE Signal Processing Magazine, vol. 24, pp. 36-46, Sep. 2007.

[17] P. Viswanath and D. N. C. Tse, "Sum capacity of the vector gaussian broadcast channel and uplink-downlink duality," IEEE Transactions on Information Theory, vol. 49, pp. 1912-1921, Aug 2003.

[18] T. L. Marzetta, "Massive MIMO: An introduction," Bell Labs Technical Journal, vol. 20, pp. 11-22, 2015.

[19] A. F. Molisch and M. Z. Win, "MIMO systems with antenna selection," IEEE microwave magazine, vol. 5, no. 1, pp. 46-56, 2004. 
[20] S. Sanayei and A. Nosratinia, "Antenna selection in MIMO systems," IEEE Communications magazine, vol. 42, no. 10, pp. 68-73, 2004.

[21] T. L. Marzetta, "Noncooperative cellular wireless with unlimited numbers of base station antennas," IEEE Transactions on Wireless Communications, vol. 9, pp. 3590-3600, November 2010.

[22] B. M. Hochwald, T. L. Marzetta, and V. Tarokh, "Multiple-antenna channel hardening and its implications for rate feedback and scheduling," IEEE transactions on Information Theory, vol. 50, no. 9, pp. 1893-1909, 2004.

[23] E. Björnson, J. Hoydis, L. Sanguinetti, et al., "Massive MIMO networks: Spectral, energy, and hardware efficiency," Foundations and Trends® in Signal Processing, vol. 11, no. 3-4, pp. 154-655, 2017.

[24] H. Q. Ngo, E. G. Larsson, and T. L. Marzetta, "Aspects of favorable propagation in massive MIMO," in 2014 22nd European Signal Processing Conference (EUSIPCO), pp. 76-80, IEEE, 2014.

[25] H. Q. Ngo, E. G. Larsson, and T. L. Marzetta, "Energy and spectral efficiency of very large multiuser MIMO systems," IEEE Transactions on Communications, vol. 61, pp. 1436-1449, April 2013.

[26] G. Americas, "LTE and 5G technologies enabling the Internet of Things," tech. rep., tech. rep., Dec, 2016.

[27] S. Borkar and H. Pande, "Application of 5G next generation network to Internet of Things," in 2016 International Conference on Internet of Things and Applications (IOTA), pp. 443-447, IEEE, 2016.

[28] M. Ibnkahla, Wireless Sensor Networks: A Cognitive Perspective. Boca Raton, FL, USA: Taylor and Francis Publishers, CRC Press, first ed., 2013.

[29] W. Wang, H. Yang, and M. Alouini, "Energy consumption for adaptive transmission over fading channels: A statistical characterization," in 2019 IEEE ICC, pp. 1-6, May 2019.

[30] D. Qiao, Y. Li, and Y. Zhang, "Adaptive modulation and power control for energy efficient video transmission over fading channels," in 2009 2nd International Congress on Image and Signal Processing, pp. 1-5, Oct 2009. 
[31] Yunxia Chen and Qing Zhao, "On the lifetime of wireless sensor networks," IEEE Communications Letters, vol. 9, pp. 976-978, Nov 2005.

[32] J. J. E. Garzás, C. B. Calzón, and A. G. Armada, "An energy-efficient adaptive modulation suitable for wireless sensor networks with SER and throughput constraints," EURASIP Journal on Wireless Communications and Networking, vol. 2007, no. 1, p. 041401, 2007.

[33] M. Soltan, I. Hwang, and M. Pedram, "Modulation-aware energy balancing in hierarchical wireless sensor networks," in 2008 3rd International Symposium on Wireless Pervasive Computing, pp. 355-359, May 2008.

[34] A. Chehri and H. Mouftah, "Energy-aware multi-hop transmission for sensor networks based on adaptive modulation," in 2010 IEEE 6th International Conference on Wireless and Mobile Computing, Networking and Communications, pp. 203-207, Oct 2010.

[35] H.-C. Yang and M.-S. Alouini, "Data-oriented wireless transmission for effective QoS provision in future wireless systems," arXiv preprint arXiv:1901.02500, 2019.

[36] H.-C. Yang and M.-S. Alouini, "Wireless transmission of big data: Data-oriented performance limits and their applications," arXiv preprint arXiv:1805.09923, 2018 .

[37] W. Wang, H. Yang, and M. Alouini, "Wireless transmission of big data: A transmission time analysis over fading channel," IEEE Transactions on Wireless Communications, vol. 17, pp. 4315-4325, July 2018.

[38] H. Yang and M. Alouini, "Characterizing energy efficiency of wireless transmission for green Internet of Things: A data-oriented approach," $\mathrm{CoRR}$, vol. abs/1805.11725, 2018.

[39] L. Lu, G. Y. Li, A. L. Swindlehurst, A. Ashikhmin, and R. Zhang, "An overview of massive MIMO: Benefits and challenges," IEEE Journal of Selected Topics in Signal Processing, vol. 8, no. 5, pp. 742-758, 2014.

[40] K. N. R. S. V. Prasad, E. Hossain, and V. K. Bhargava, "Energy efficiency in massive MIMO-based 5G networks: Opportunities and challenges," IEEE Wireless Communications, vol. 24, no. 3, pp. 86-94, 2017. 
[41] C. Sun, C. She, and C. Yang, "Energy-efficient resource allocation for ultrareliable and low-latency communications," in GLOBECOM 2017 - 2017 IEEE Global Communications Conference, pp. 1-6, 2017.

[42] E. Björnson, L. Sanguinetti, J. Hoydis, and M. Debbah, "Optimal Design of Energy-Efficient Multi-User MIMO Systems:Is Massive MIMO the Answer?," IEEE Transactions on Wireless Communications, vol. 14, pp. 3059-3075, June 2015.

[43] M. M. Hossain, C. Cavdar, E. Björnson, and R. Jäntti, "Energy saving game for Massive MIMO: Coping with daily load variation," IEEE Trans. on Vehicular Tech., vol. 67, pp. 2301-2313, March 2018.

[44] K. Senel, E. Björnson, and E. G. Larsson, "Adapting the number of antennas and power to traffic load: When to turn on massive MIMO?," in 2018 IEEE Wireless Communications and Networking Conference (WCNC), pp. 1-6, April 2018.

[45] J. Hoydis, S. ten Brink, and M. Debbah, "Massive MIMO in the UL/DL of cellular networks: How many antennas do we need?," IEEE Journal on Selected Areas in Com., vol. 31, pp. 160-171, Feb 2013.

[46] B. M. Lee, "Improved energy efficiency of massive MIMO-OFDM in batterylimited IoT networks," IEEE Access, vol. 6, pp. 38147-38160, 2018.

[47] N. R. S. V. P. Koppisetti, S. Mallick, and V. K. Bhargava, "Design of adaptive antenna systems for LTE using genetic algorithm and particle swarm optimization," in 2015 IEEE 28th Canadian Conference on Electrical and Computer Engineering (CCECE), pp. 1054-1059, 2015.

[48] B. Martinez, M. Montón, I. Vilajosana, and J. D. Prades, "The power of models: Modeling power consumption for IoT devices," IEEE Sensors Journal, vol. 15, pp. 5777-5789, Oct 2015.

[49] H. Jayakumar, K. Lee, W. S. Lee, A. Raha, Y. Kim, and V. Raghunathan, "Powering the Internet of Things," in 2014 IEEE/ACM International Symposium on Low Power Electronics and Design (ISLPED), pp. 375-380, Aug 2014.

[50] M. Ibnkahla, Adaptive Signal Processing in Wireless Communications. Boca Raton, FL, USA: CRC Press, 1 ed., 2008. 
[51] A. J. Goldsmith and Soon-Ghee Chua, "Variable-rate variable-power MQAM for fading channels," IEEE Transactions on Communications, vol. 45, pp. 1218 1230, Oct 1997.

[52] M.-S. Alouini and A. J. Goldsmith, "Adaptive modulation over Nakagami fading channels," Kluwer J. Wireless Communications, vol. 13, pp. 119-143, May 2000.

[53] S. U. Pillai, T. Suel, and Seunghun Cha, "The perron-frobenius theorem: some of its applications," IEEE Signal Processing Magazine, vol. 22, pp. 62-75, March 2005. 